\title{
Engaging Users: The Future of Academic Library Web Sites
}

\section{Shu Liu}

This article examines current academic library Web site practices and recommends a conceptual model for future academic library Web site design. The author investigated $111 \mathrm{ARL}$ member library Web sites and has summarized current site content, design patterns, and innovative features. The author discusses the Web 2.0 principles as defined by Tim O'Reilly and reviews the current literature on Web 2.0 implications for library Web services. The author proposes making the academic library Web site a virtual place and recommends a conceptual model to inform future academic library Web site design based on the investigation and the discussion. Future academic library Web sites might feature any of the following: 1) user focus, 2) personalization, 3) user engagement, 4) online communities, and 5) remixability.

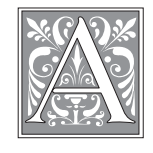

cademic library Web sites are libraries' virtual presentation to the world. Beyond providing information about libraries and library services, academic library Web sites provide access to online catalogs, electronic databases, subject resources, library instruction/tutorials, and digital collections. In alignment with each institution's mission, academic library Web sites are gateways to information that supports faculty and student research and educational needs. Currently, most academic library Web sites are inventories and access points for such information. As the massive growth of Internet resources and new searching and sharing tools such as Google, Amazon, eBay, uTube, Flickr, del.icio.us, and MySpace give users more power, ease, and fun in information seeking, academic library Web sites face stiff competition in the area of user preferences, despite the fact that academic library Web sites provide considerably higher quality and better scholarly information. As Brian Detlor and Vivian Lewis point out, "Academic libraries are at a turning point." ${ }^{1}$ Chuck Thomas and Robert H. McDonald state, "Libraries are facing a new generation of online users who are technologically savvy and integrate information access and use in all spheres of their lives to an unprecedented degree. They approach the traditional library with certain expectation that may conflict with the existing services, policies, and values of the library as information broker." 2 Thomas Frey, a senior futurist at the DaVinci Institute, argues, "... the library of the future will need to be designed to accommodate the changing needs of its constituency." 3

"Web 2.0" was introduced to the library world a couple of years ago. The concept

Shu Liu is Digital Content Librarian in the Libraries at Colorado State University; e-mail: shu. liu@colostate.edu. 
of Web 2.0 began with a conference brainstorming session between O'Reilly Media and MediaLive International in 2004. According to Tim O'Reilly, Web 2.0 is a set of key principles demonstrating successful features of a new era of Internet companies/applications. These key principles include: ${ }^{4}$

- the Web as platform

- harnessing collective intelligence

- data is the next Intel inside ("Intel" is used here as a metaphor to emphasize the importance of data)

- end of the software release cycle

- lightweight programming models

- software above the level of a single device

- rich user experiences

O'Reilly himself later provided a compact definition of Web 2.0, stating: "Web 2.0 is the business revolution in the computer industry caused by the move to the internet as platform, and an attempt to understand the rules for success on that new platform. Chief among those rules is this: Build applications that harness network effects to get better the more people use them." ${ }^{5}$

In this article, I make recommendations for future academic library Web site design, inspired by the Web 2.0 key principles, particularly, the Web as platform, harnessing collective intelligence, lightweight programming models, and rich user experiences. These recommendations include the following:

- provide an efficient, flexible, and user-oriented interface

- build a virtual space that facilitates rich user experiences

- engage users and encourage collective intelligence

- support content sharing and nurture online communities

By implementing these recommendations, academic library Web sites will streamline and enrich users' experiences in interacting with and utilizing information throughout their learning and research activities and will keep up with rapid changes in user behaviors. I used the following methodology to arrive at these recommendations. I chose to investigate the Web sites of the Association of Research Libraries (ARL) members ${ }^{6}$ to discover the current state of academic library Web sites. ARL members are a well-defined set of academic libraries and include my own institution, Colorado State University Libraries. My discoveries have been documented in an appendix and I will summarize current content, design patterns, and innovative features of those Web sites in this paper. I will then discuss the key principles as defined by Tim O'Reilly and review the current literature on Web 2.0 implications for library Web services. Based on my investigation and discussion, I will provide a conceptual model echoing the above-listed recommendations to inform future Web site design for academic libraries.

\section{Current State of Academic Library Web Sites}

This investigation was limited to those ARL member Web sites specifically affiliated with a college or university and provided in the English language; the sample consisted of a total number of 111 ARL members. This investigation focused on content and design patterns and was, in most cases, limited to library homepages. My discoveries are detailed in Appendix 1. Use of specific examples is not intended to be pejorative or critical of specific library Web pages in part or in whole; rather, the examples are used to provide comparison among the various pages, and a certain amount of subjectivity is therefore unavoidable.

Detlor and Lewis previously evaluated 107 ARL member Web sites to assess and measure library Web site functionality in a quantitative manner. They used a codebook derived from the published literature that was dedicated to the design and usability of library Web sites and other related electronic interfaces. They made recommendations surrounding a central argument, "academic libraries build robust library Web 
sites to combat increasing competition from commercial services." ${ }^{7}$ Although I acknowledge Detlor and Lewis' findings and insights, the investigation for this paper differs from theirs in approach and comprehensiveness. I used a snapshot of current academic library homepages' content and design as a basis for my recommendations. A summary of the investigation appears below in the categories of content, design patterns, and innovative features.

\section{Content}

The following major content elements were identified on the library homepages:

- search/find resources by format

- resources by subject/subject guides

- research assistance/how do I.../ help

- about (library)/library information

- library services

- search site, and/or site A-Z

- ask a librarian/ask us

- news/announcements/events

- contact us/suggestions

The following content elements were less common but frequently appeared:

- library account(s)

- digital collections/Web exhibits

- portals by user types such as students, faculty, staff, alumni, friends, and visitors

In general, the homepage content is focused on and arranged according to library functions, resources, and services. Online library information and resources are vast and comprehensive, requiring multiple searching and browsing pathways. Content typically follows a "one-design-for-all" approach and there are few customization features and few opportunities for users to create, publish, and exchange content.

\section{Design Patterns}

The following design patterns appeared on the library homepages:
- columns by category

- horizontal sections by category

- mixed columns and horizontal sections

- four equally divided sections with sidebar(s)

- mouse-over links with sidebar(s)

Most library homepages have an overwhelming amount of text and links. A few academic libraries are less text and link focused by employing a more graphical design. ${ }^{8}$

\section{Innovative Features}

About thirty libraries employed RSS (Really Simple Syndication) ${ }^{9}$ for library news and events (see Appendix 2).

Four library Web sites provide personalized library spaces, named "My Library," "My Personal Library," or "My Search Space," aggregating into one spot access to library user accounts, course reserve materials, library alerts, databases, citation tools, and/or search preferences/ results. ${ }^{10}$ On nine library homepages, links are provided to a university-level Web portal or centralized Web service. ${ }^{11}$

Ten library homepages provide a "Google Scholar" search box or a direct link to search Google Scholar. ${ }^{12}$ Two library homepages also provide a link to search "Windows Live Academic."13

Live Chat, as a reference communication method, appears on almost all library homepages. The table below illustrates other unique and user-friendly innovative features.

\section{Web 2.0 Principles and Implications}

Some Web 2.0 transformations, according to O'Reilly, include the evolutions from Britannica Online to Wikipedia, from personal Web sites to blogging, from publishing to participation, from content management systems to wikis, from directories (taxonomy) to tagging ("folksonomy" - general users' classifications of Internet resources), and from stickiness (static Web pages, manual update) to syndication (dynamic Web pages, auto update). ${ }^{14}$ 
The Future of Academic Library Web Sites 9

\begin{tabular}{|c|c|}
\hline \multicolumn{2}{|c|}{$\begin{array}{l}\text { TABLE } 1 \\
\text { Innovative Features Discovered from } 111 \text { Academic Library Web Sites }\end{array}$} \\
\hline \multicolumn{2}{|l|}{ A. Use of "Web 2.0" Tools } \\
\hline RSS for new library resources & $\begin{array}{l}\text { MIT, U. of Oklahoma, U. of Saskatchewan, U. } \\
\text { of Toronto, York U. }\end{array}$ \\
\hline $\mathrm{B} \log s$ & $\begin{array}{l}\text { Brigham Young U., U. of Minnesota, North } \\
\text { Carolina State, Temple, Texas A\&M, Washing- } \\
\text { ton U. in St. Louis }\end{array}$ \\
\hline Wikis & $\begin{array}{l}\text { Colorado State (not linked from homepage), } \\
\text { North Carolina State }\end{array}$ \\
\hline Podcasts & $\begin{array}{l}\text { Arizona State ("The Library Channel"), MIT } \\
\text { (coming later), Ohio State }\end{array}$ \\
\hline Personal Bookmarks/Tagging & U. of Pennsylvania, U. of Saskatchewan \\
\hline \multicolumn{2}{|l|}{ B. User Engagement } \\
\hline "Rate this page" & U. of Toronto \\
\hline $\begin{array}{l}\text { "The Library Video (created by stu- } \\
\text { dents, for students!)" }\end{array}$ & U. of Virginia \\
\hline $\begin{array}{l}\text { "What Students are Saying about } \\
\text { LIBR1100 ..." }\end{array}$ & Texas Tech \\
\hline "Question of the Week" & Boston College \\
\hline \multicolumn{2}{|l|}{ C. Homepage Customization } \\
\hline "Customize this site for ..." & U. of Connecticut \\
\hline \multicolumn{2}{|l|}{ D. Aggregated Resources } \\
\hline "Knowledge Bank" (IR model: DSpace) & Ohio State \\
\hline $\begin{array}{l}\text { "The Teaching Library" (for faculty and } \\
\text { librarians to teach information literacy) }\end{array}$ & U. of Tennessee \\
\hline "InfoTree" (subject resources) & Ohio U. \\
\hline "Knowledge Base" (library information) & U. of Oklahoma \\
\hline "Library Survival Guide" (instructions) & York U. \\
\hline \multicolumn{2}{|l|}{ E. Single Sign-on } \\
\hline "Passport York" & York U. \\
\hline \multicolumn{2}{|l|}{ F. Recommending Information } \\
\hline "Popular Links" side bar & U. of Toronto \\
\hline "Top 10 Databases" & U. of Wisconsin-Madison \\
\hline "Featured item" & Vanderbilt U. \\
\hline "Resource Spotlight" & Brown U. \\
\hline "Featured Resources" & U. at Buffalo (SUNY), U. of California at Davis \\
\hline \multicolumn{2}{|l|}{ G. Multimedia } \\
\hline Library Virtual Tour & Texas Tech, U. of Oklahoma \\
\hline Audio Tours, Library Tutorial Videos & Arizona State \\
\hline \multicolumn{2}{|l|}{ H. Use of Familiar Format } \\
\hline Library hours in a calendar & Texas Tech \\
\hline
\end{tabular}




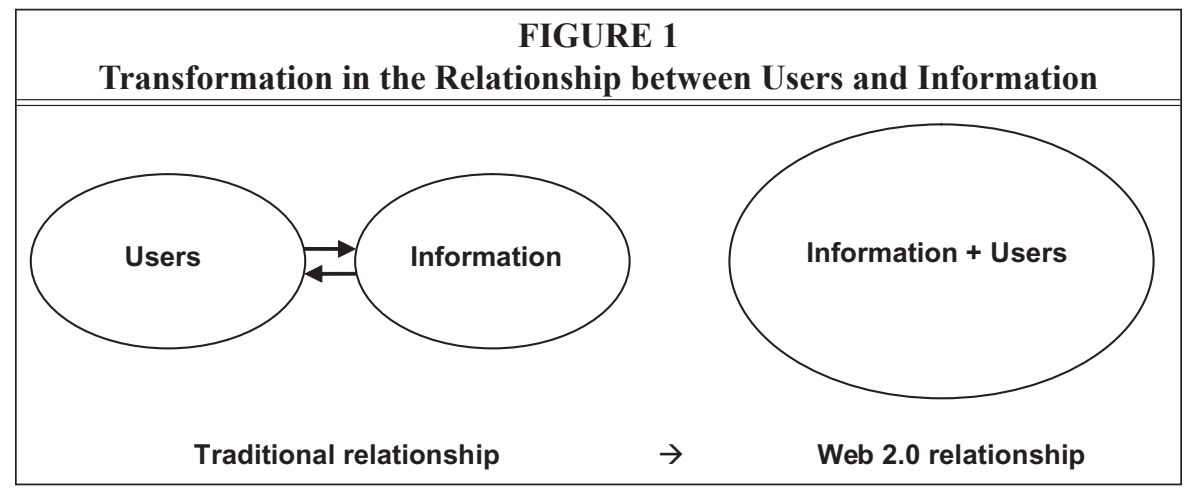

As the "read/write Web, " the "collaborative Web," or the "expressive Web," Web 2.0 is an "organic" information environment where user engagement adds value. Web 2.0 tools "set inclusive defaults for aggregating user data and building value as a side effect of ordinary use of the application." ${ }^{15}$ The more users participate, the more value a service provides: users connect with, inspire, help, and improve one another through the service's participation mechanisms; the service's user-generated content is then available over the network for reuse. In the Web 2.0 era, the relationship between users and information is transformed from stand-alone, separate silos to mutually inclusive, mutually reliant, and reciprocal action-and-reaction entities. The diagram below illustrates this point.

The Web 2.0 culture also includes syndication of dynamic content and lightweight program/service models to create usable and exciting innovations called "mashups." Mashups challenge the imaginations of information designers and providers to create and deliver more usable tools and better services over the Internet. As O'Reilly states: "think syndication, not coordination"; "innovation [is] in assembly." 16 Relating to recognition of the idea (or already a fact?) that, in the Web 2.0 era, users and information are integrated, I consider it legitimate to say "Web 2.0 = users + mashups." O'Reilly's Web 2.0 application examples include Google, eBay, Amazon, Wikipedia, blog- ging, RSS, del.icio.us ${ }^{17}$ (a social bookmarking Web site), Flicker ${ }^{18}$ (a photo sharing Web site), iTunes ${ }^{19}$ (a digital media player by Apple), $\mathrm{TiVo}^{20}$ (a popular brand of digital video recorder in the United States), and $\mathrm{AJAX}^{21}$ (a programming technique for creating interactive Web applications).

Several librarians and information researchers have recognized the implications of Web 2.0 principles on library Web services. Karen Coombs, Head of Web Services at the University of Houston Libraries, proposed four principles to construct a concept of "Library Virtual Presence in 2010": "1. A library's virtual presence will be more than the library's web site. 2. A library's web site will be responsive to the needs of users. 3 . Through a comprehensive effort in digital collection building, the library's virtual presence will exploit unique materials in its collections. 4. Users will be able to come to the site not only to search for information but also to collaborate and share ideas." ${ }^{22}$ Doug Achterman, the library media teacher at San Benito High School in Hollister, California, discussed the use of blogs and wikis (the most popular applications of Web 2.0) in a school setting to promote school library programs, advance instructional practice in collaboration with classroom teachers, and connect with students. ${ }^{23}$ Elizabeth Yakel, Associate Professor at the School of Information, University of Michigan, investigated ways in which Web 2.0 features are being used in archival access 
systems (such as live chat reference, tagging/commenting, bookbags/shopping carts, and recommender systems/ratings) and how more might be incorporated in the future. ${ }^{24}$ Jack M. Maness, Librarian/ Computer Science Bibliographer at the University of Colorado Boulder Libraries, addressed how Web 2.0 technologies (such as synchronous messaging, streaming media, blogs, wikis, social networks, tagging, RSS feeds, and mashups) might intimate changes in how libraries provide access to their collections and user support. ${ }^{25}$ Edward J. Maloney, Director of Research and Learning Technologies at Georgetown University, discussed the challenges that Web 2.0 poses to e-learning technologies in higher education and proposed a Digital Notebook project as one model to "incorporate the paradigm-altering technologies of Web 2.0 into teaching and learning." ${ }^{26}$ In addition, University of Houston Libraries Strategic Directions (2006-2010) declared "expand our virtual presence" as their first strategic principle and "include content on the Libraries' web site that is interactive, engaging, and participatory" in their course of action. ${ }^{27}$

\section{Recommendations}

The core value of libraries is the service they provide to their users. The ultimate goal of academic library Web sites is to make preselected and preserved information readily available to users for their educational and research needs. The investigation described at the beginning of this article reveals that current academic library Web sites have made significant efforts and are successful in putting available information online and providing users with complete access to online library resources and services. Several academic library Web sites have employed innovative features that bring users closer to what libraries have to offer and encourage some level of user engagement (see table 1). However, the universe of information presented on academic library homepages still focuses on library functions, requires numerous pathways for access, has overwhelming options, and takes a "one-design-for-all" approach that fails to recognize users as individuals. In addition, few current academic library Web sites offer opportunities for users to create and share user-generated content.

\begin{tabular}{|l|}
\hline \multicolumn{1}{|c|}{ FIGURE 2 } \\
Conceptual Model: Part 1 - Library Homepage \\
\hline \hline \begin{tabular}{|l|l|}
\hline [Library Banner] \\
[Global Navigation: possibility - direct links to library resources by subject, \\
list most popular subjects and use "more..." to link to a complete subject list]
\end{tabular} \\
\begin{tabular}{|l|l|l}
\hline $\begin{array}{l}\text { [User type] } \\
\text { Undergraduate }\end{array}$ & $\begin{array}{l}\text { [Quick Link] } \\
\text { Login to My } \\
\text { Library Space } \\
\text { [User type] } \\
\text { Faculty \& Staff } \\
\text { Top news } \\
\text { Top used } \\
\text { resources ... } \\
\text { Search ... }\end{array}$ \\
\hline $\begin{array}{ll}\text { [User type] } \\
\text { Graduate Students }\end{array}$ & $\begin{array}{l}\text { [User type] } \\
\text { Friends \& Visitors }\end{array}$ \\
\hline
\end{tabular}
\end{tabular}




\section{FIGURE 3}

Conceptual Model: Part 2 - Portal Page for Undergraduate Students

[Library Banner]

[Global Navigation: possibility - direct links to library resources by subject, list most popular subjects and use "more..." to link to a complete subject list]

[Text] Welcome, [university name] Undergraduate Students!

Library Research

- What you need to know about Library Research [Information Literacy]

- $\quad$ Explore library resources [list]

- I need help! [FAQ and contacts]

\section{Internet Research}

- What you need to know about Internet Research [Information Literacy]

- Explore Internet resources [list]

- I need help! [FAQ and contacts]

"Discovery Channel"

[Learn about new research tools/strategies]

\section{Login to My Library Space}

Email: [entry box]

Password: [entry box]

Login [Button] Create! [Button]

I forgot my password! [Link]

Featured Library Spaces

[Thumbnails below]

Featured undergraduate student spaces

Featured graduate student spaces

Featured faculty spaces

Featured community spaces
To capitalize on the insights of a variety of information professionals on the Web 2.0 culture, and to take action on these observations, I propose making the academic library Web site a virtual place and recommend a three-part conceptual model described in details below. Before beginning, I would like to note that the suggested model only demonstrates what users might want. Technological details, usability testing, and privacy/liability issues need to be further explored beyond this paper.

\section{Library Homepage}

Reduce the intimidating appearance of library homepages' by using as little text and linking as possible to convey only the most necessary access points and by employing an appealing graphical design that accommodates usability and accessibility requirements. I suggest using a portal approach where users first identify themselves as belonging to one of several general groups (for example, graduate students or undergraduate students). Each group-specific portal would then lead a user to a specific set of resources customized for the group. Label each portal with a graphic or a color. Figure 2 illustrates the first part of the conceptual model and provides an example of a library homepage (graphics and colors are not applied in this illustration).

\section{Library Portal Page}

Once a group-specific portal page is selected, a user may navigate among a customized set of resources or may choose to create or $\log$ in to a personal library space. Figure 3 illustrates the second part of the conceptual model and provides an example of a library portal page for the user group of undergraduate students.

Content in the left main body is adjusted accordingly. For example, on a portal page for the user group of faculty and staff, that portion of the page could be a list of 
links to the library's catalog or the library's federated searching system (ideally, federated searching is an aggregated search of all library resources such as catalogs, research databases, digital collections, and so on), an e-journals list, a databases list, a scholarly Internet resources list, a scholarly publishers list, a subject librarians list, and the "Discovery Channel" (that is, a themed section for users to learn about new research tools/strategies).

To create a personal library space, a user would need to enter basic, required information such as name, e-mail, and user type. Once a user account is created and authentication is completed, a user may then create a profile to list optional information such as college, department, major, year in school, specialization, research interests, personal interests, and "What I most use the library for..."

\section{"My Library Space"}

My Library Space is a one-stop information environment for an individual user and provides a combined set of information technology tools for use. A user may customize the interface and select what tools/links to use/display in the space, according to personal need. Within My Library Space, a user can save and access preferred library resources, interact with others using preferred tools (such as instant messaging, e-mail, and bulletin board), work with data/documentation online, create and share content (using blog, wiki, or podcast), and integrate school/work activities. A user may wish to create a personal library space for either or both of the rich user experiences and the "one-stop-shopping" capabilities it provides. Figure 4 illustrates the last part of the conceptual model and provides an

\section{FIGURE 4}

\section{Conceptual Model: Part 3 - My Library Space}

[Library Banner]

[Global Navigation: possibility - direct links to library resources by subject, list most popular subjects and use "more..." to link to a complete subject list]

[Text] Welcome, XXX [a user's first name]!

Now XX users online; my friends: $\mathrm{xxx}, \mathrm{xxx}, \mathrm{xxx}, \mathrm{xxx} \ldots$ [click a name to open online chat]

My Favorite Library Tools/Resources

Customize my space [link or button]

My Saved Searches

My Library Collections [use tagging here]

My Multimedia [multimedia tools and resources]

My Online Classes [link to $\mathrm{LMS}^{1}$ ]

My Work Space [work with data/documents online]

My School Calendar

My School Email

My Portfolio

My Events

My Bulletin Board

My Published Space
My Blogs
My Wiki Space
My RSS List
My Podcasts
My Tags
Friends'Spaces
New Spaces ${ }^{3}$

${ }^{1}$ Learning Management System, such as WebCT, Blackboard.

${ }^{2}$ a digital media file, or a series of such files, that is distributed over the Internet using syndication feeds for playback on portable media players and personal computers, see http://en.wikipedia. org/wiki/Podcast.

${ }^{3}$ newly published spaces by other library users. 
example of a My Library Space, regardless of user type.

This conceptual model demonstrates ways in which Web 2.0 principles might be incorporated into future academic library Web site design. The recommended design will provide an efficient, flexible, and user-oriented interface and thus minimize the distance between users and library resources/services. This effort will require collaborative experimentation among librarians and information technology professionals. The back-end work will be extensive, demanding continuous creativity and exploration. Meanwhile, the convenience and rich experiences we offer users will be very rewarding.

Based on this conceptual model, future academic library web sites might feature:

1. User focus: focus on library users by presenting library resources in a targeted and customized manner.

2. Personalization: recognize library users as individuals by giving them opportunities to configure their own library interfaces and to select tools and content based on personal needs.

3. User engagement: provide sufficient tools to allow and encourage library users in content creation and exchange.

4. Online communities: nurture the development of online communities by connecting individuals through online publishing and sharing tools such as blogs, wikis, podcasting, and tagging.

5. Remixability: employ a mashup ap- proach to aggregate current and emerging information technologies to provide library users with opportunities to explore new possibilities to discover and work with information resources.

\section{Conclusion}

Rachel Singer Gordon states, "L2 (Library 2.0) recognizes that each library implements technology and services in ways appropriate to its community. We all start from different points and have different needs. Embrace this one-size-doesn'tfit-all approach." ${ }^{28}$ In conclusion, future academic library Web sites should:

- switch the focus from presenting information arranged according to library functions and resources to providing targeted and customizable tools and services to library users;

- design a seamless, one-stop information environment that minimizes users' way-finding effort and nurtures the formation and dissemination of knowledge, ideas, and experiences; and

- respond to users' changing needs and give users opportunities to express, share, and learn.

The OCLC College Students' Perceptions of Libraries and Information Resources: A Report to the OCLC Membership (2005) pointed out that "It is time to rejuvenate the 'Library' brand." ${ }^{29}$ The opportunity to be responsive to the changing information environment and rejuvenate academic library Web sites is now in our hands.

\section{Notes}

1. Brian Detlor and Vivian Lewis, "Academic Library Web Sites: Current Practice and Future Directions," Journal of Academic Librarianship 32, no. 3 (2006): 251-58.

2. Chuck Thomas and Robert H. McDonald, "Millennial Net Value(s): Disconnects Between Libraries and the Information Age Mindset" (Aug. 15, 2005), Florida State University D-Scholarship Repository, Article \#4. Available online at http://dscholarship.lib.fsu.edu/general/4/. [Accessed 23 July 2007].

3. Thomas Frey, "The Future of Libraries: Beginning the Great Transformation." Available online at http://www.davinciinstitute.com/page.php?ID=120. [Accessed 23 July 2007].

4. Tim O'Reilly, "What Is Web 2.0: Design Patterns and Business Models for the Next Generation of Software" (2005). Available online at www.oreillynet.com/pub/a/oreilly/tim/ news/2005/09/30/what-is-web-20.html. [Accessed 23 July 2007].

5. Tim O'Reilly, "Web 2.0 Compact Definition: Trying Again" (2006). Available online at http://radar.oreilly.com/archives/2006/12/web_20_compact.html. [Accessed 23 July 2007]. 
The Future of Academic Library Web Sites 15

6. ARL member libraries. Available online at www.arl.org/members.html. [Accessed 4 December 2006].

7. Detlor and Lewis, "Academic Library Web Sites," 251-58.

8. University of British Columbia, University of California at Los Angeles, Columbia University, Iowa State University, University of Southern California, and Washington State University.

9. See the Wikipedia entry on RSS. Available online at http://en.wikipedia.org/wiki/RSS_ (file_format). [Accessed 23 July 2007].

10. University of California at Davis, University of Iowa, North Carolina State University, and University of Toronto.

11. University of Arizona, University of British Columbia, McGill University, Louisiana State University, Texas A\&M University, University of Utah, Virginia Tech, Wayne State University, and Washington State University.

12. University of Alabama, Brigham Young University, Colorado State University, Florida State University, University of Georgia, Howard University, University of North Carolina at Chapel Hill, University of Texas at Austin, Texas A\&M University, and Wayne State University; Google Scholar search engine: Available online at http://scholar.google.com/ [Accessed 23 July 2007].

13. Texas A\&M University and Florida State University; Windows Live Academic search engine: Available online at http://academic.live.com/ [Accessed 23 July 2007].

14. O'Reilly, "What Is Web 2.0"

15. Ibid.

16. Ibid

17. Available online at http://del.icio.us/. [Accessed 23 July 2007].

18. Available online at www.flickr.com/. [Accessed 23 July 2007].

19. Available online at www.apple.com/itunes/. [Accessed 23 July 2007].

20. Information available online at www.tivo.com/. [Accessed 23 July 2007].

21. Jesse James Garrett, "Ajax: A New Approach to Web Applications," Adaptive Path (Feb. 18, 2005). Available online at http://adaptivepath.com/publications/essays/archives/000385.php. [Accessed 23 July 2007].

22. Karen Coombs, "Planning for Now and Then," Library Journal 131 (2006): 2-3.

23. Doug Achterman, "Making Connections with Blogs and Wikis," CSLA Journal 30, no. 1 (2006): 29-31.

24. Elizabeth Yakel, "Inviting the User into the Virtual Archives," OCLC Systems \& Services 22, no. 3 (2006): 159-63.

25. Jack M. Maness, "Library 2.0 Theory: Web 2.0 and Its Implications for Libraries," Webology 3, no. 2 (2006): Article 25. Available online at www.webology.ir/2006/v3n2/a25.html. [Accessed 23 July 2007].

26. Edward J. Maloney, "What Web 2.0 Can Teach Us about Learning," The Chronicle of Higher Education 53, no. 18 (2007): B26-27.

27. University of Houston Libraries, “UH Libraries Strategic Directions, 2006-2010,” (July 2006). Available online at http://info.lib.uh.edu/strategic_directions.pdf. [Accessed 23 July 2007].

28. Rachel Singer Gordon, "What Will You Do Today?" Library Journal 131, no. 17 (2006): 43.

29. OCLC, "College Students' Perceptions of Libraries and Information Resources: A Report to the OCLC Membership" (2005). Available online at www.oclc.org/reports/perceptionscollege. htm. [Accessed 23 July 2007]. 


\begin{tabular}{|c|c|c|c|c|c|c|c|c|c|c|}
\hline હ્ને & 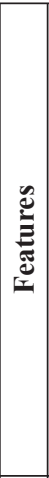 & $\begin{array}{l}\frac{1}{3} \\
0 \\
0 \\
0 \\
0 \\
0 \\
0 \\
00 \\
0 \\
0 \\
0\end{array}$ & $\begin{array}{l}n \\
0 \\
0 \\
z \\
\infty \\
\tilde{n} \\
\simeq 1 \\
\end{array}$ & $\begin{array}{l}\tilde{n} \\
\tilde{j} \\
\tilde{z} \\
\tilde{n} \\
\tilde{n} \\
\end{array}$ & 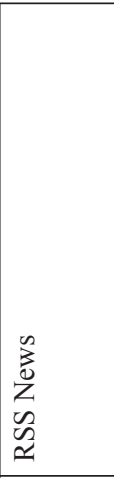 & 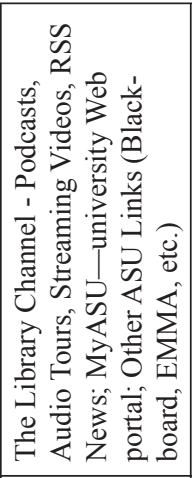 & & 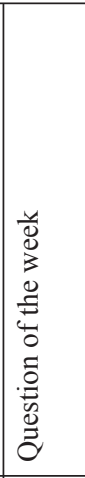 & & 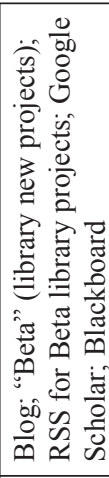 \\
\hline 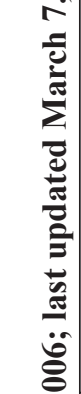 & 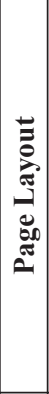 & 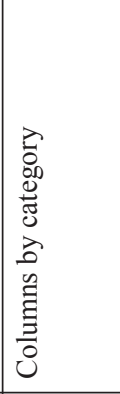 & 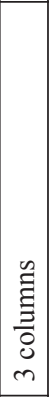 & 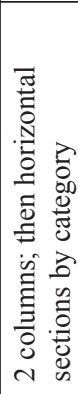 & 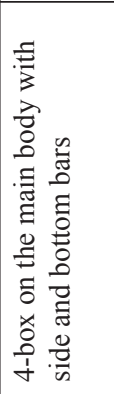 & 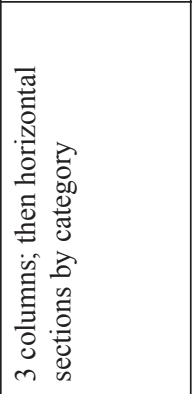 & 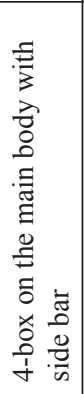 & 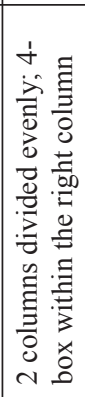 & 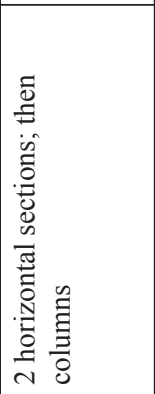 & 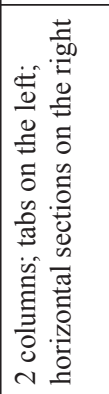 \\
\hline 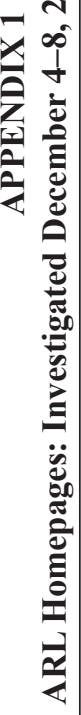 & 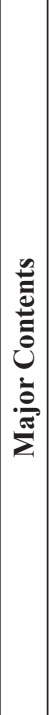 & 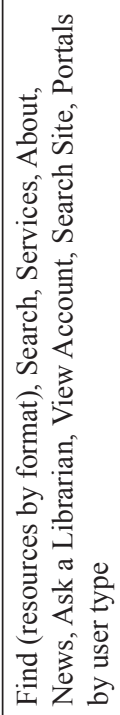 & 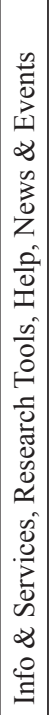 & 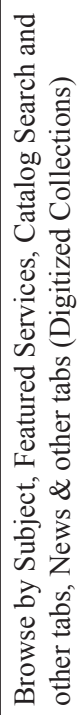 & 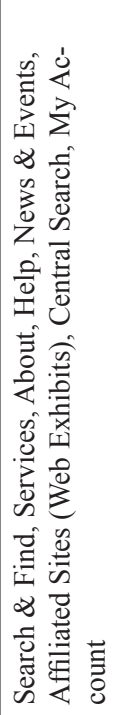 & 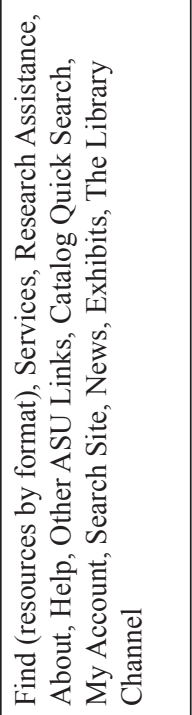 & 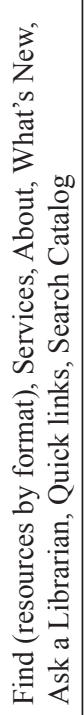 & 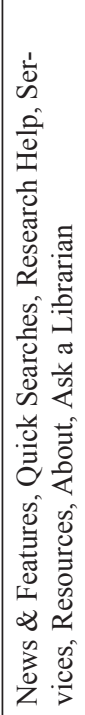 & 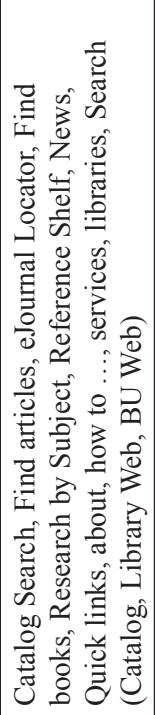 & 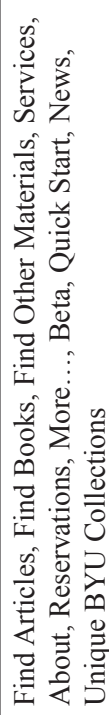 \\
\hline & 䏤 & 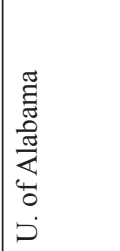 & 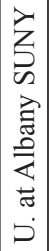 & 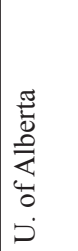 & 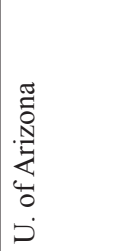 & 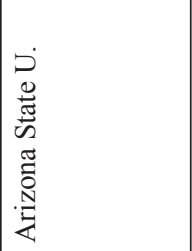 & 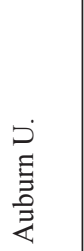 & $\mid \begin{array}{l}8 \\
\stackrel{8}{0} \\
\stackrel{0}{\overline{0}} \\
0 \\
0 \\
0 \\
0 \\
0 \\
0\end{array}$ & 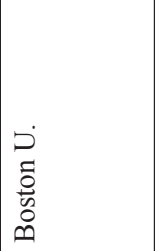 & 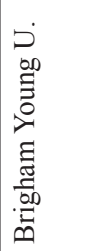 \\
\hline & $\mathbf{z}$ & - & $\sim$ & $m$ & $\nabla$ & $n$ & 0 & $r$ & $\infty$ & a \\
\hline
\end{tabular}


The Future of Academic Library Web Sites 17

\begin{tabular}{|c|c|c|c|c|c|c|c|c|c|}
\hline 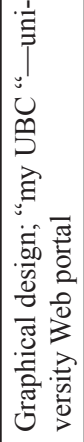 & 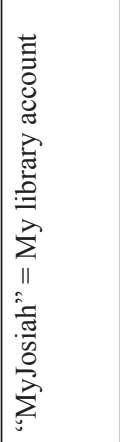 & $\begin{array}{l}n \\
\hat{z} \\
z \\
n \\
n \\
\tilde{n}\end{array}$ & & 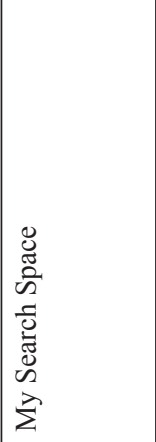 & 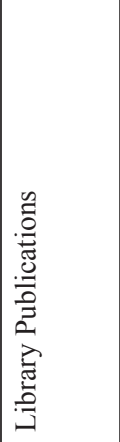 & 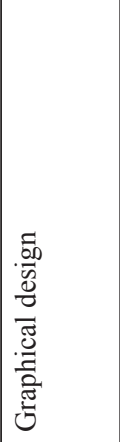 & $\begin{array}{l}n \\
z \\
z \\
n \\
n \\
01 \\
\approx\end{array}$ & & \\
\hline 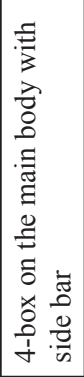 & 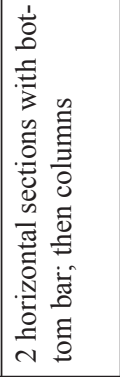 & 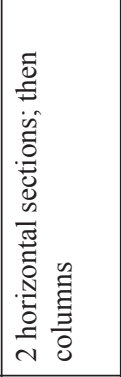 & 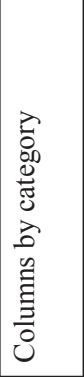 & 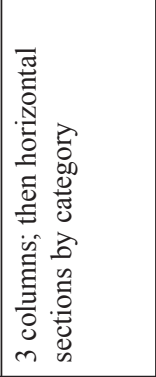 & 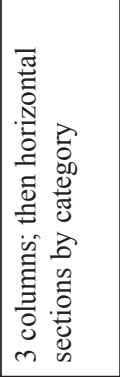 & 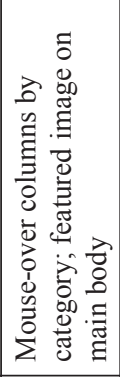 & 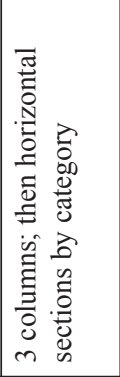 & 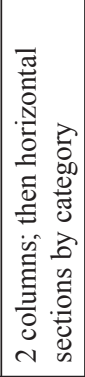 & 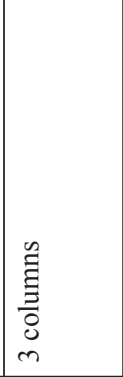 \\
\hline 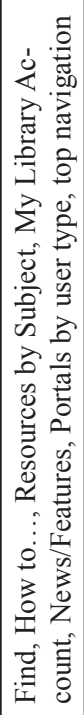 & 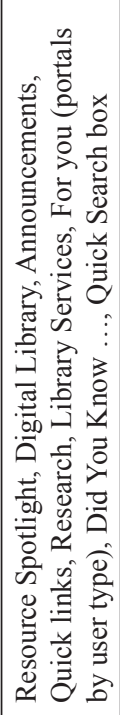 & 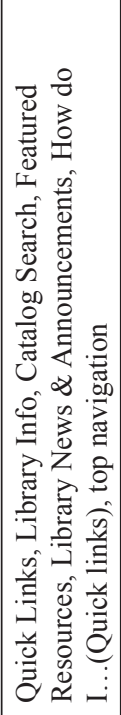 & 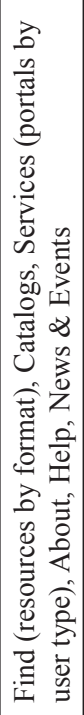 & 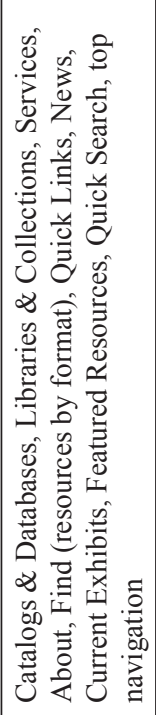 & 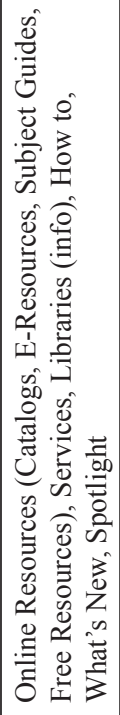 & 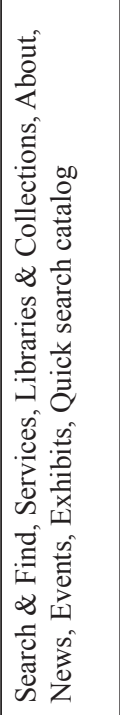 & 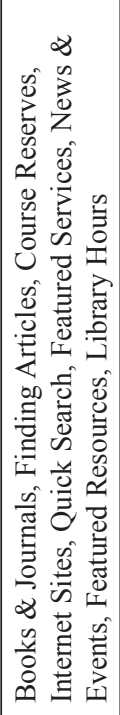 & 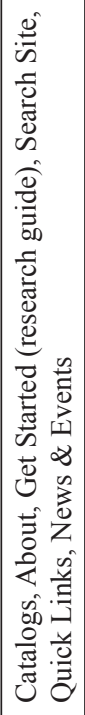 & 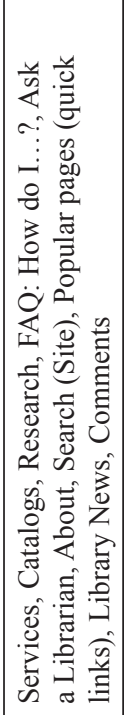 \\
\hline 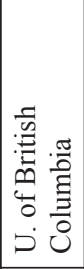 & D & 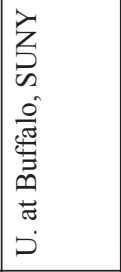 & 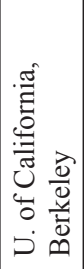 & 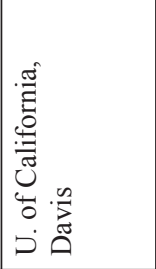 & 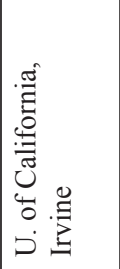 & 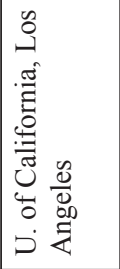 & 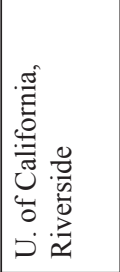 & 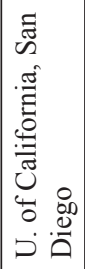 & 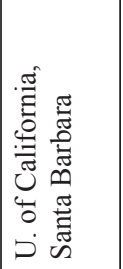 \\
\hline 으 & $=$ & 2 & 2 & 士 & $n$ & $=$ & $=$ & $\infty$ & 2 \\
\hline
\end{tabular}




\begin{tabular}{|c|c|c|c|c|c|c|c|}
\hline & & 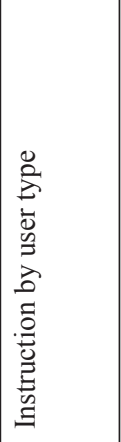 & & & 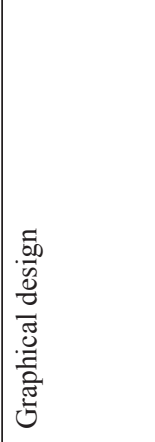 & 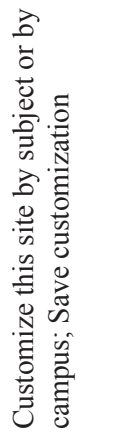 & \\
\hline 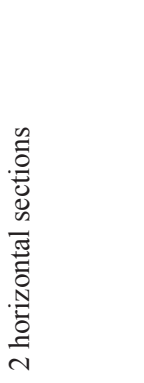 & 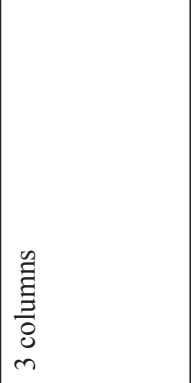 & 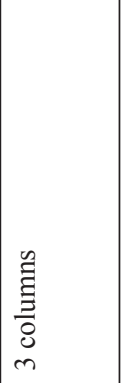 & 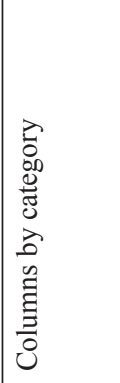 & 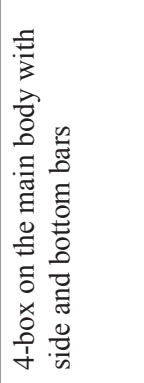 & 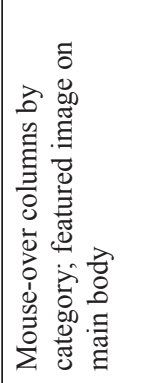 & 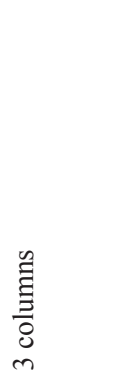 & 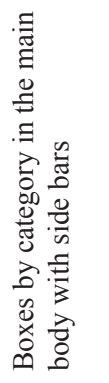 \\
\hline 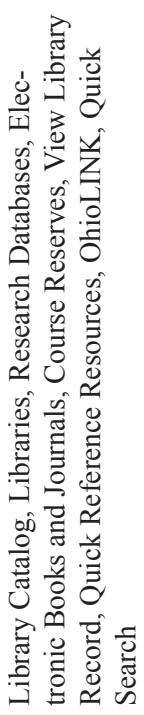 & 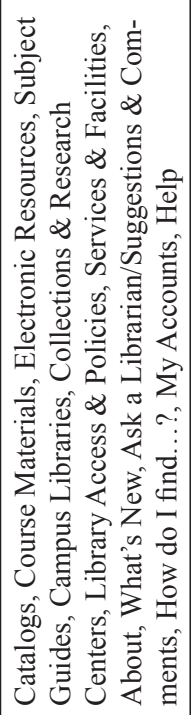 & 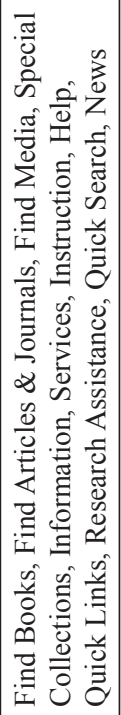 & 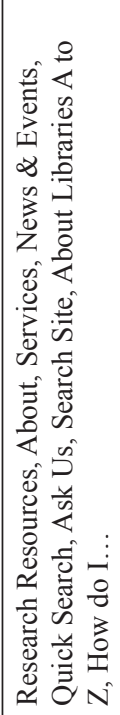 & 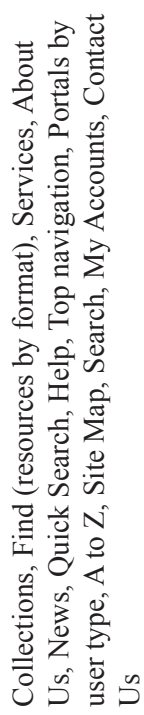 & 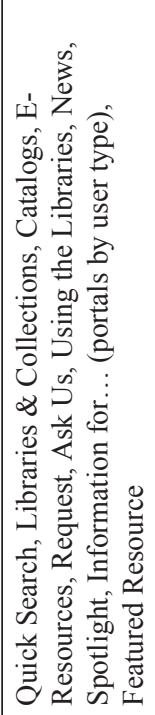 & 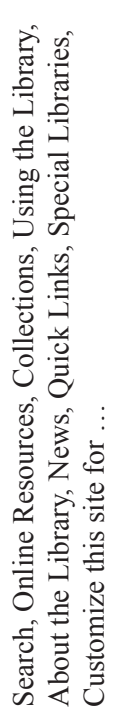 & 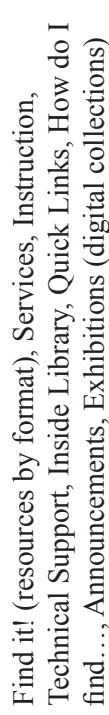 \\
\hline 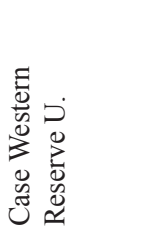 & 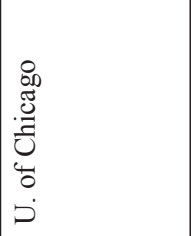 & 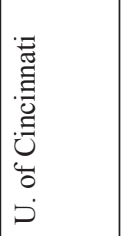 & 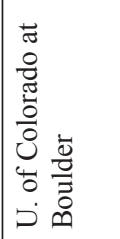 & 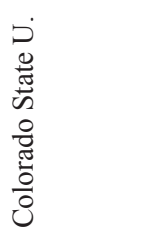 & 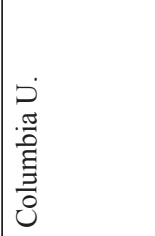 & 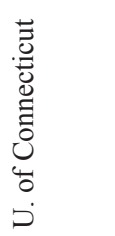 & $\begin{array}{l}\stackrel{D}{\bar{D}} \\
\bar{\Xi} \\
\dot{\Xi}\end{array}$ \\
\hline N & $\vec{\sim}$ & $\mathbb{N}$ & $\tilde{\sim}$ & $\stackrel{\sim}{\sim}$ & $\stackrel{2}{a}$ & $\stackrel{\circ}{\sim}$ & $\hat{\imath}$ \\
\hline
\end{tabular}


The Future of Academic Library Web Sites 19

\begin{tabular}{|c|c|c|c|c|c|c|c|c|c|}
\hline 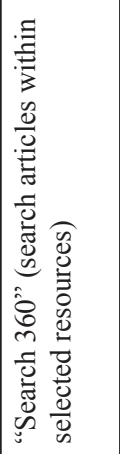 & & & & & 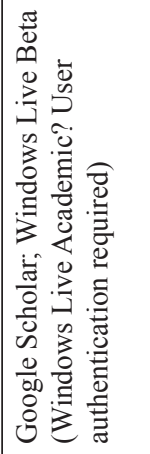 & & & $\begin{array}{l}\frac{1}{3} \\
0 \\
\frac{1}{0} \\
\mathscr{2} \\
0 \\
00 \\
0 \\
0 \\
0\end{array}$ & \\
\hline 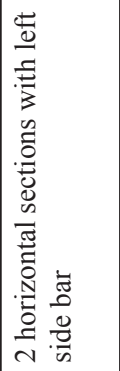 & 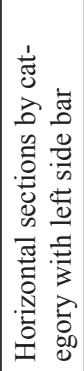 & 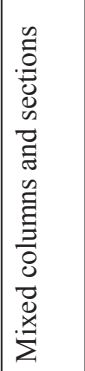 & 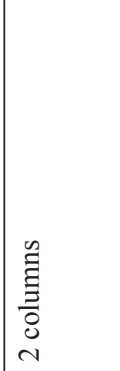 & 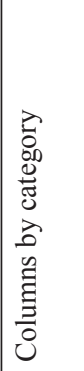 & 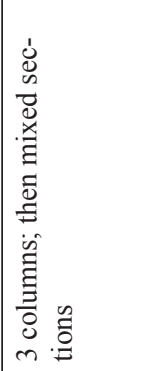 & 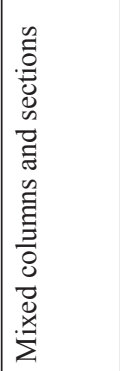 & 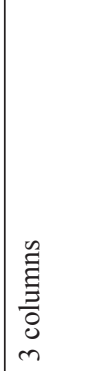 & 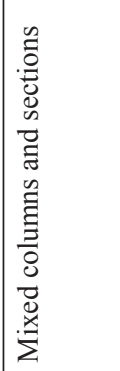 & 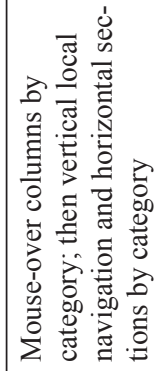 \\
\hline 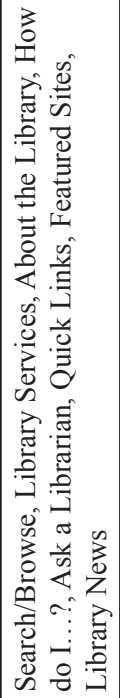 & 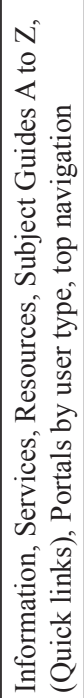 & 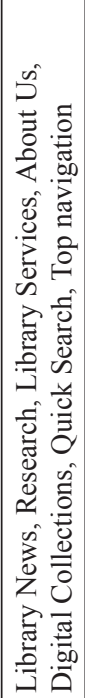 & 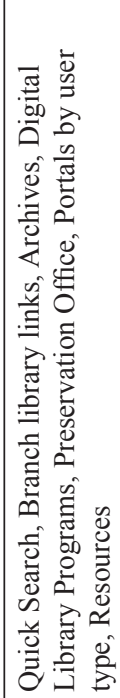 & 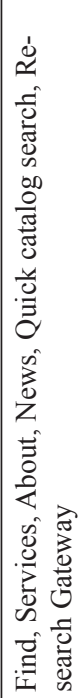 & 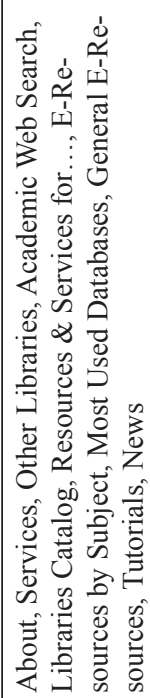 & 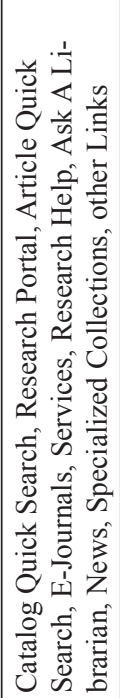 & 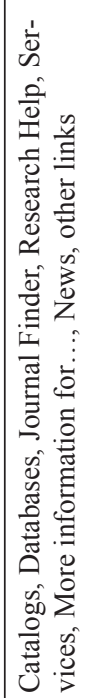 & 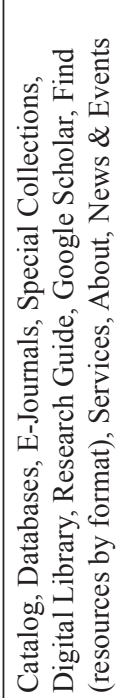 & 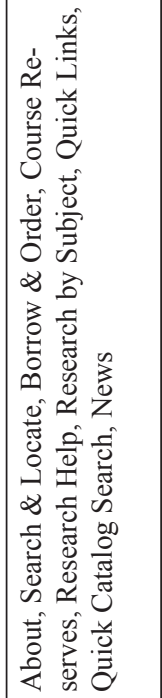 \\
\hline 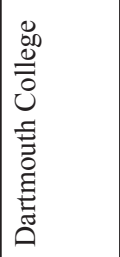 & 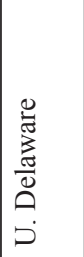 & $\begin{array}{l}D \\
0 \\
\frac{y}{\vec{D}} \\
0\end{array}$ & 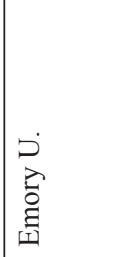 & 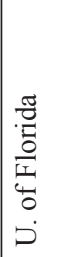 & 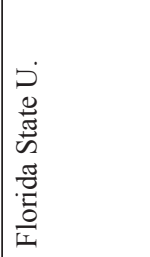 & 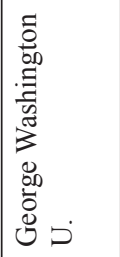 & 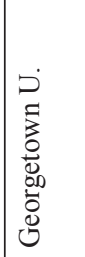 & 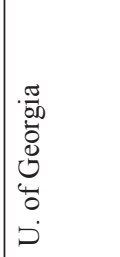 & 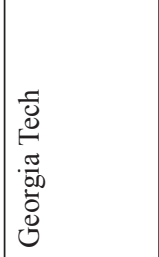 \\
\hline$\stackrel{\infty}{\sim}$ & i & $m$ & $m$ & $\approx$ & m & m & $m$ & in & m \\
\hline
\end{tabular}




\begin{tabular}{|c|c|c|c|c|c|c|c|c|c|c|c|c|}
\hline 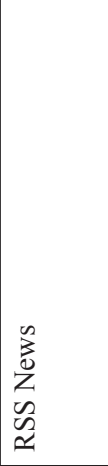 & & & 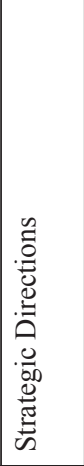 & 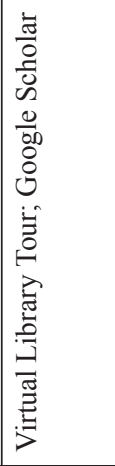 & & & & 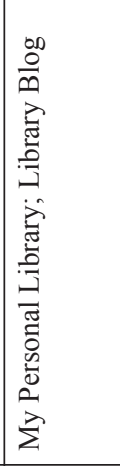 & 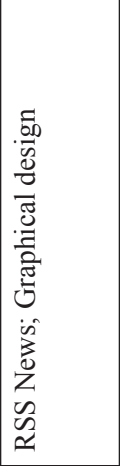 & & & 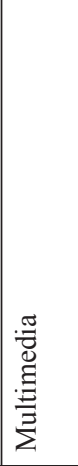 \\
\hline 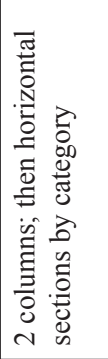 & 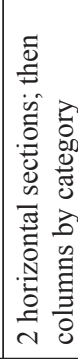 & \begin{tabular}{|c}
$y$ \\
$\vdots$ \\
$\vdots$ \\
0 \\
0 \\
$m$ \\
$m$
\end{tabular} & 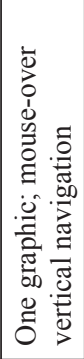 & 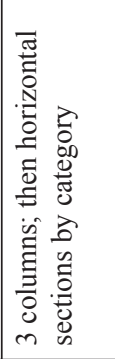 & 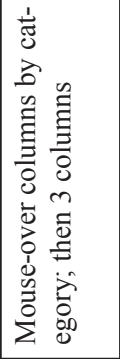 & 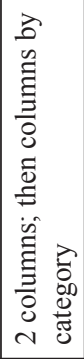 & 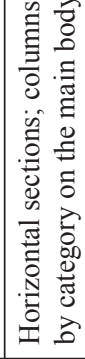 & 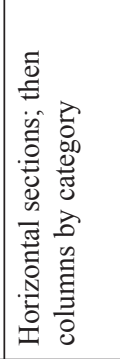 & 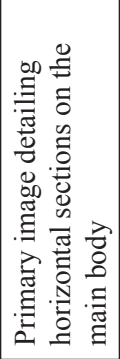 & 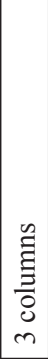 & 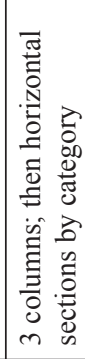 & 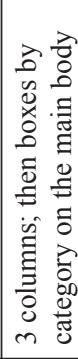 \\
\hline 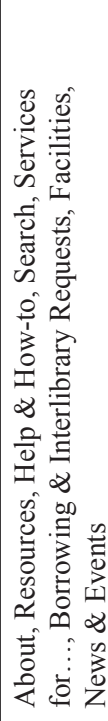 & 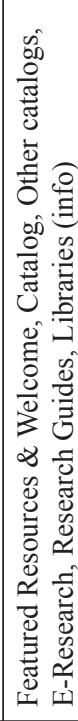 & 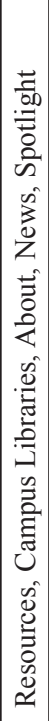 & 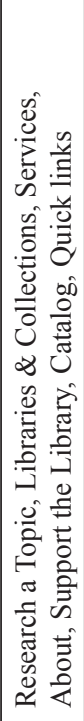 & 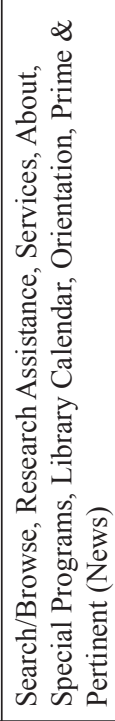 & 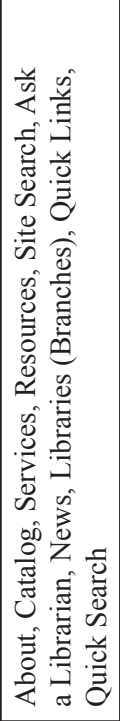 & 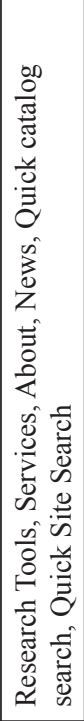 & 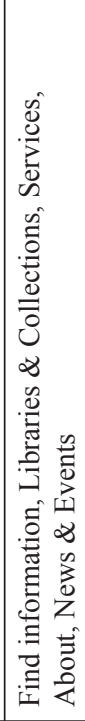 & 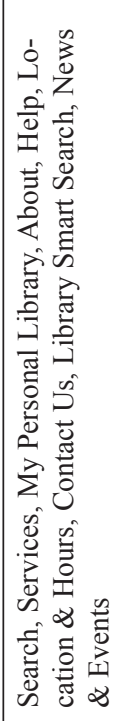 & 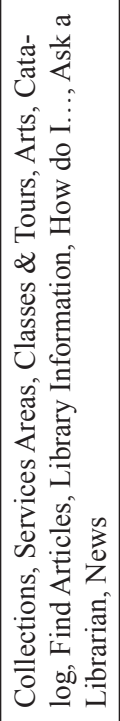 & 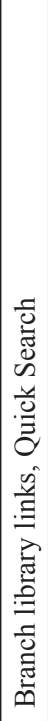 & 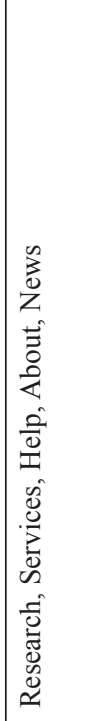 & 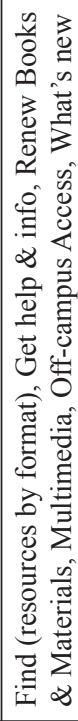 \\
\hline 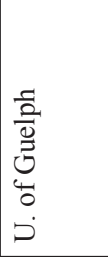 & 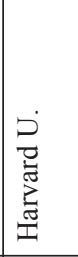 & 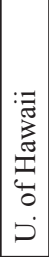 & \begin{tabular}{|l}
0 \\
0 \\
0 \\
0 \\
0 \\
01 \\
4 \\
0 \\
0 \\
$\dot{10}$
\end{tabular} & $\begin{array}{l}0 \\
0 \\
0 \\
0 \\
0 \\
0 \\
0 \\
0\end{array}$ & 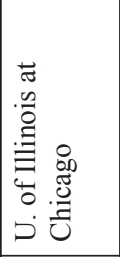 & 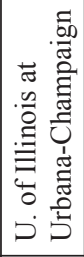 & 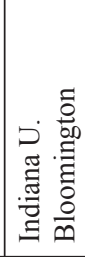 & 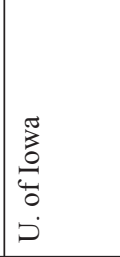 & 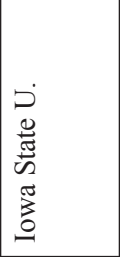 & $\begin{array}{l}0 \\
0 \\
.3 \\
0 \\
0 \\
0 \\
01 \\
0 \\
0 \\
0 \\
0 \\
0\end{array}$ & 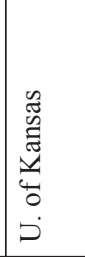 & 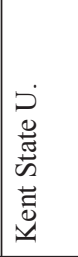 \\
\hline$m$ & $\stackrel{\rho}{\circ}$ & f & $\gamma$ & F & $\forall$ & f & $\forall$ & F & $\gamma$ & $\stackrel{\infty}{+}$ & $\stackrel{q}{q}$ & in \\
\hline
\end{tabular}


The Future of Academic Library Web Sites 21

\begin{tabular}{|c|c|c|c|c|c|c|c|c|c|c|c|}
\hline 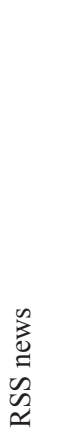 & 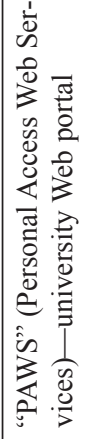 & & 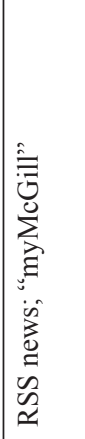 & 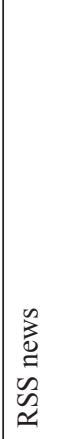 & & 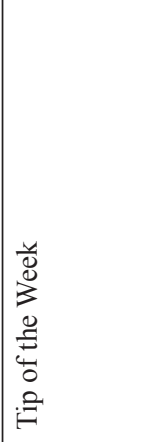 & 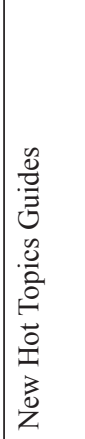 & 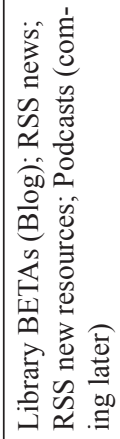 & & 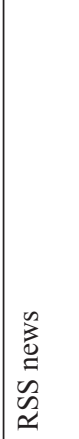 & 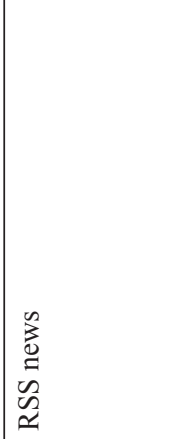 \\
\hline 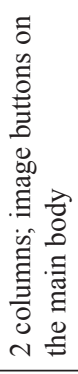 & 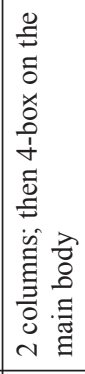 & 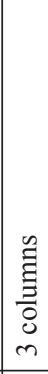 & 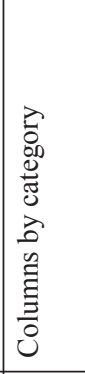 & 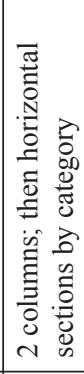 & & 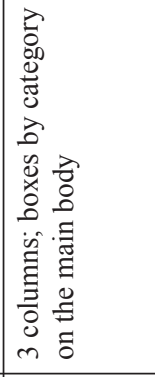 & 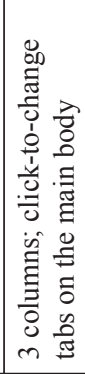 & 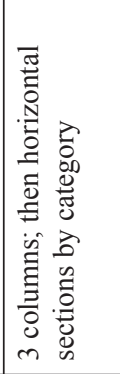 & 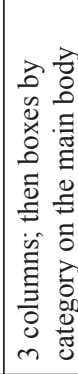 & 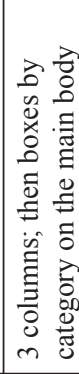 & 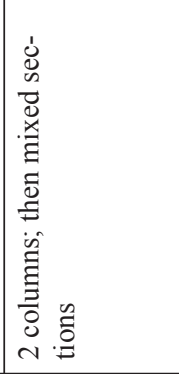 \\
\hline 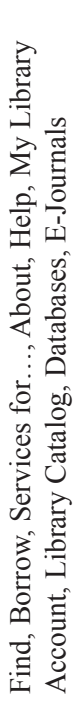 & 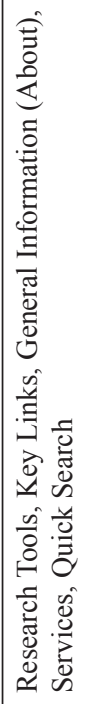 & 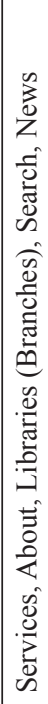 & 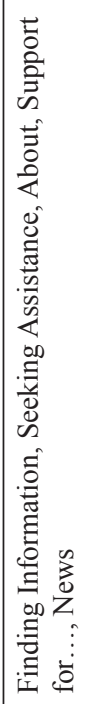 & 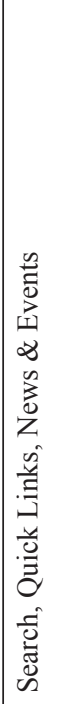 & 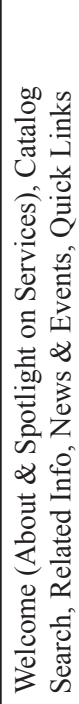 & 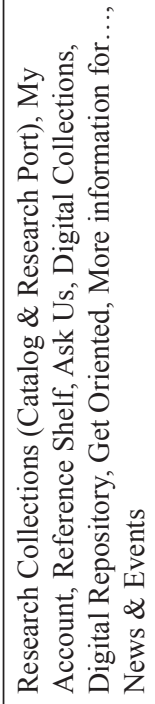 & 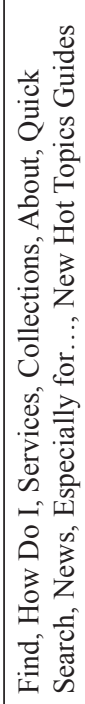 & 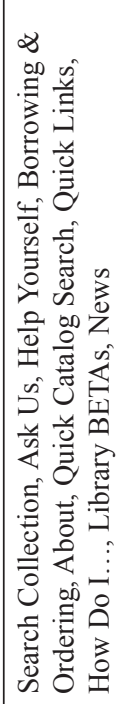 & 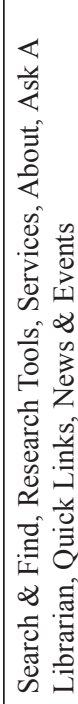 & 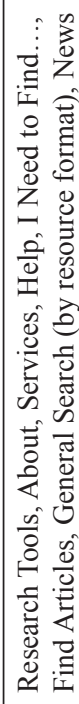 & 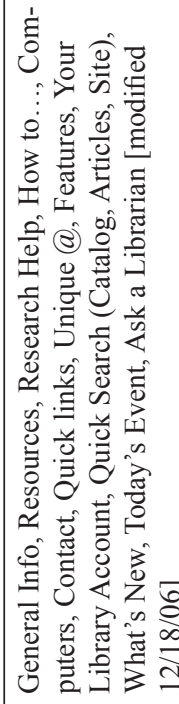 \\
\hline 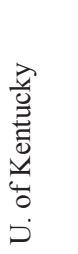 & 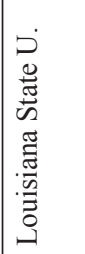 & 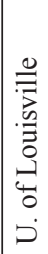 & 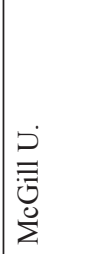 & 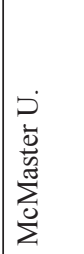 & 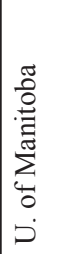 & 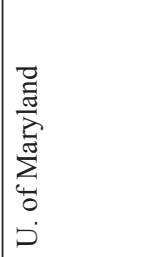 & 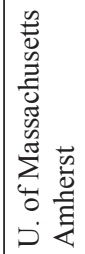 & $\stackrel{\Xi}{\Sigma}$ & 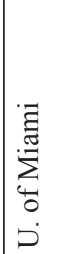 & 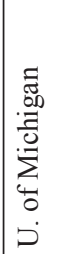 & 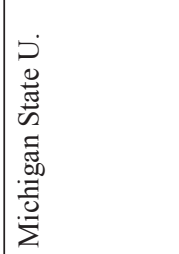 \\
\hline in & $\tilde{n}$ & $n$ & in & $n$ & $\ddot{n}$ & $\hat{n}$ & in & in & 8 & $\overrightarrow{6}$ & $\widetilde{\sigma}$ \\
\hline
\end{tabular}




\begin{tabular}{|c|c|c|c|c|c|c|c|c|c|}
\hline 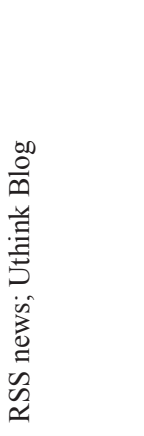 & & & & & 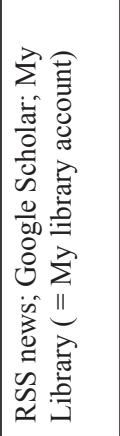 & 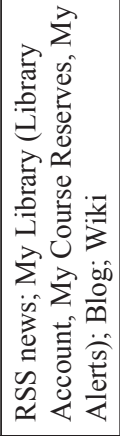 & 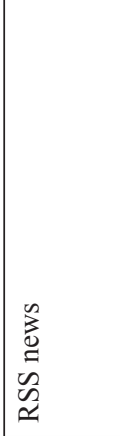 & & 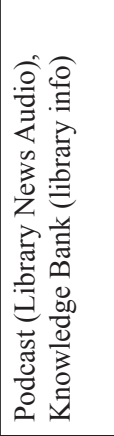 \\
\hline 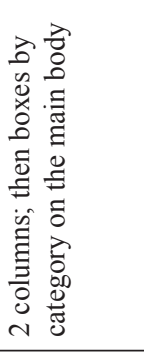 & 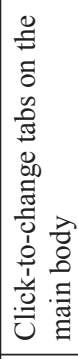 & 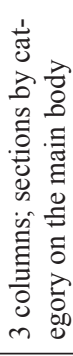 & 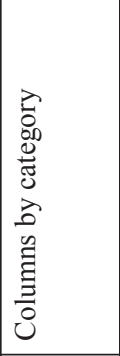 & 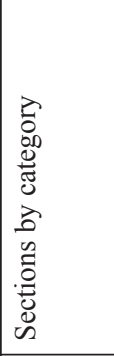 & 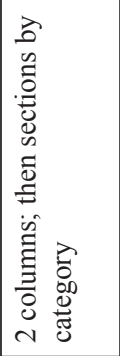 & 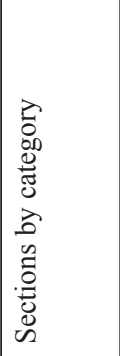 & 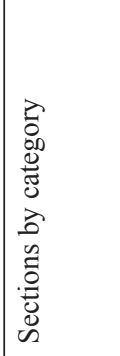 & 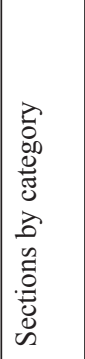 & 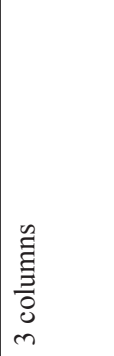 \\
\hline 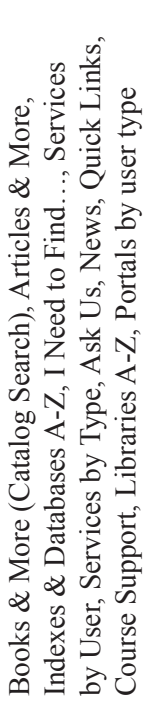 & 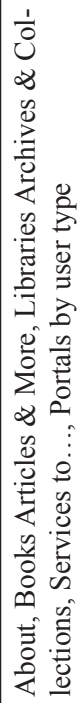 & 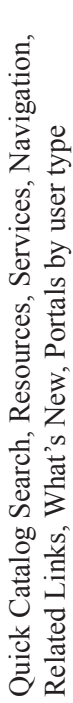 & 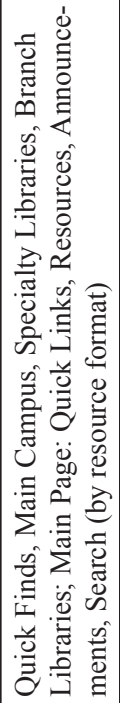 & 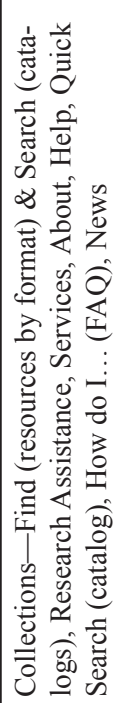 & 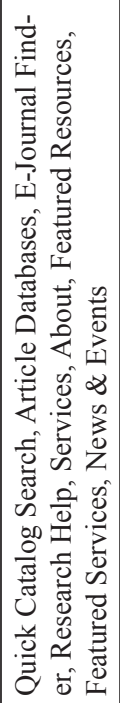 & 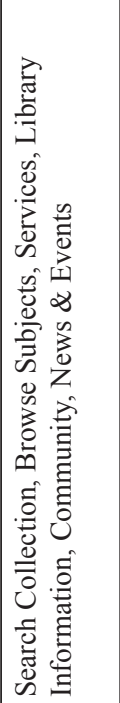 & 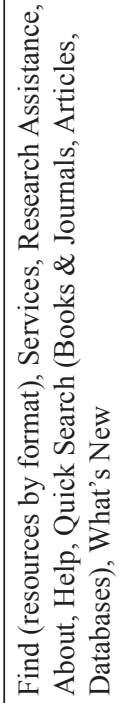 & 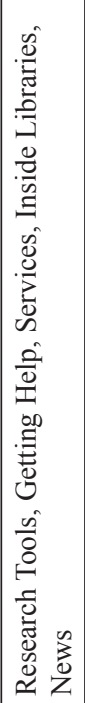 & 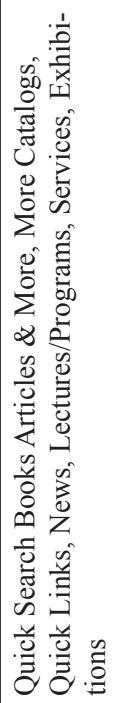 \\
\hline 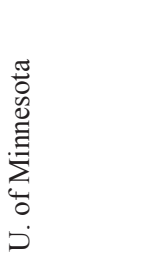 & 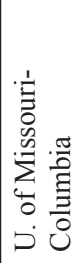 & 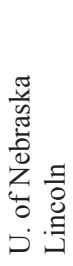 & 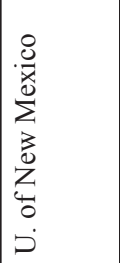 & 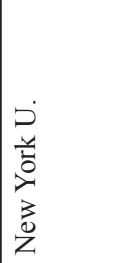 & 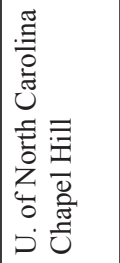 & 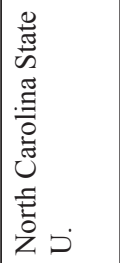 & 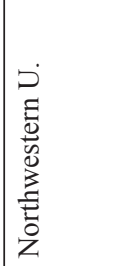 & 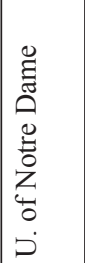 & 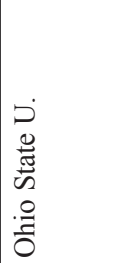 \\
\hline 6 & t & 6 & 8 & $\hat{\sigma}$ & $\infty$ & 8 & i? & $F$ & $\mathbb{N}$ \\
\hline
\end{tabular}


The Future of Academic Library Web Sites 23

\begin{tabular}{|c|c|c|c|c|c|c|c|c|c|c|c|}
\hline 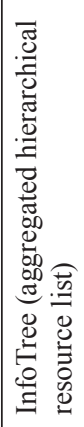 & 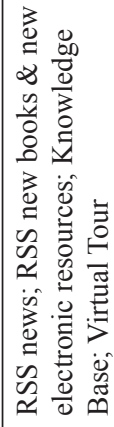 & & & 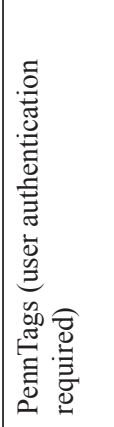 & & & 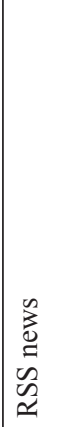 & & 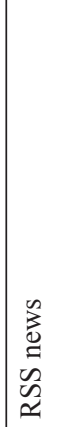 & & \\
\hline 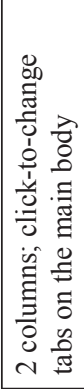 & 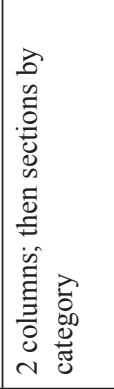 & 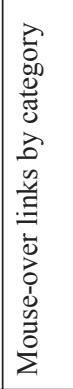 & 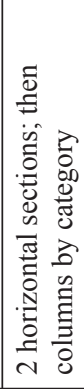 & 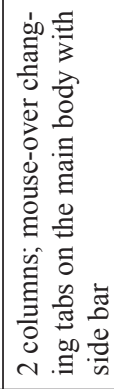 & 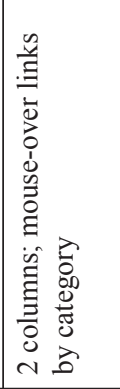 & 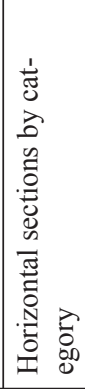 & 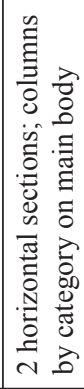 & 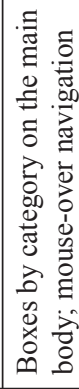 & 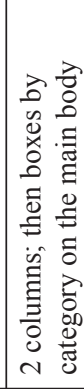 & 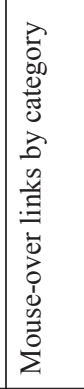 & 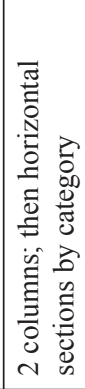 \\
\hline 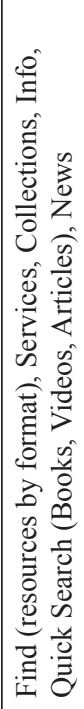 & 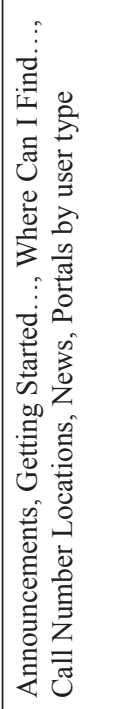 & 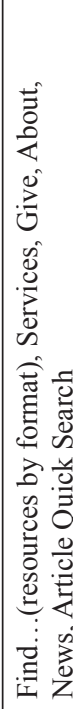 & 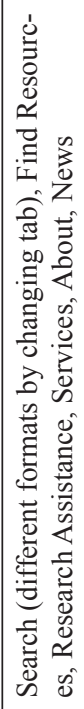 & 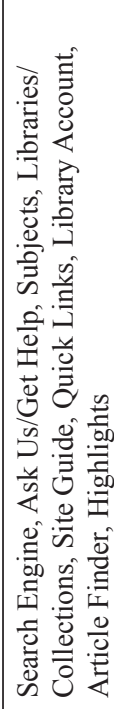 & 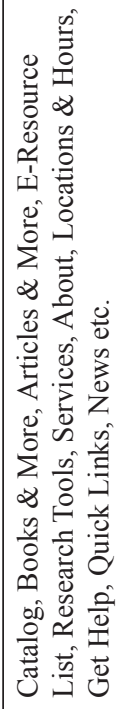 & 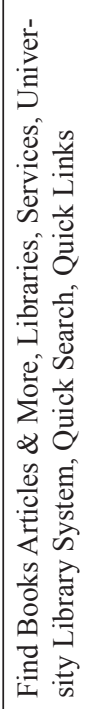 & 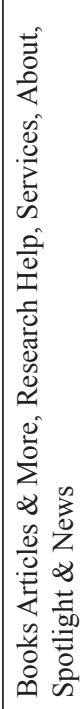 & 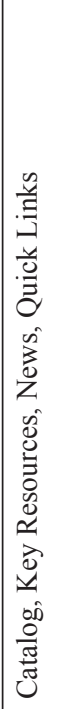 & 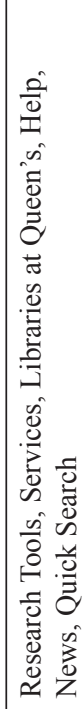 & 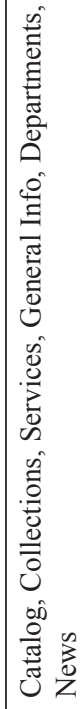 & 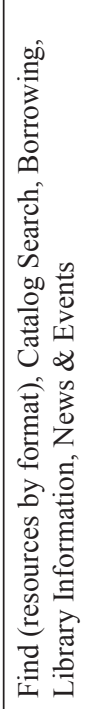 \\
\hline 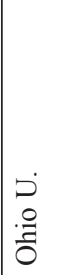 & 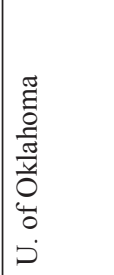 & 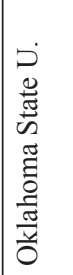 & 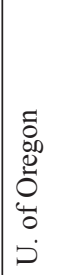 & 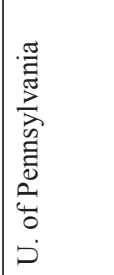 & 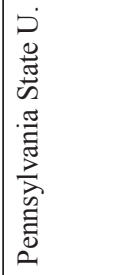 & 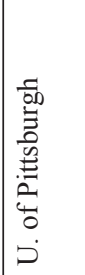 & 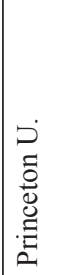 & 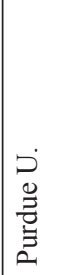 & $\mid \begin{array}{l}D \\
\infty \\
\tilde{\Xi} \\
\stackrel{\Xi}{\Xi} \\
\tilde{\Xi}\end{array}$ & 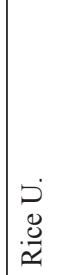 & 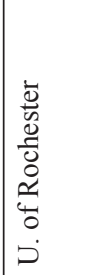 \\
\hline$\cong$ & $r$ & $r$ & 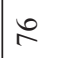 & 2 & $\stackrel{\infty}{\sim}$ & $\pi$ & $\infty$ & $\infty$ & $\infty$ & $\infty$ & $\infty_{\infty}^{+}$ \\
\hline
\end{tabular}




\begin{tabular}{|c|c|c|c|c|c|c|c|c|c|}
\hline & 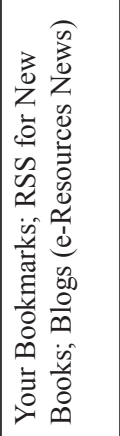 & & 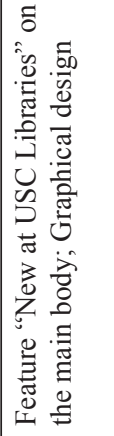 & 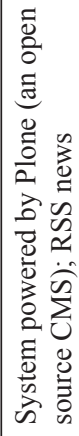 & 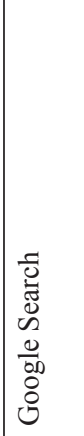 & & $\begin{array}{l}00 \\
0 \\
0 \\
0 \\
0 \\
0 \\
0 \\
00 \\
0 \\
0 \\
0 \\
0 \\
0 \\
0 \\
0 \\
0 \\
0 \\
0 \\
0 \\
0 \\
2 \\
0 \\
0 \\
0 \\
0 \\
z\end{array}$ & 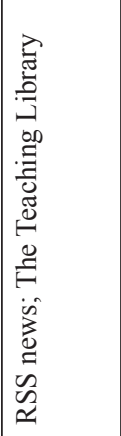 & 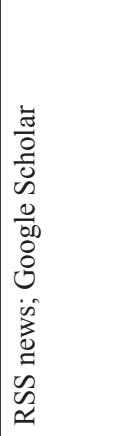 \\
\hline 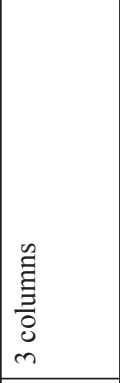 & 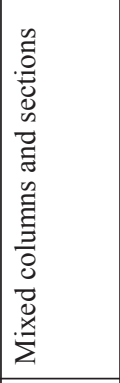 & 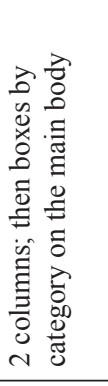 & 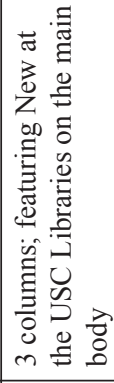 & 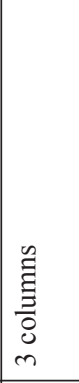 & 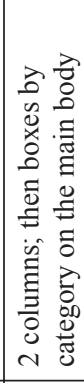 & 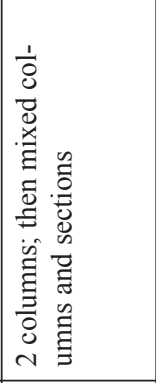 & 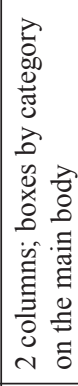 & 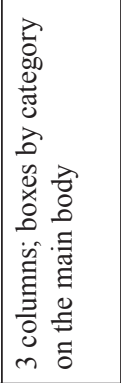 & 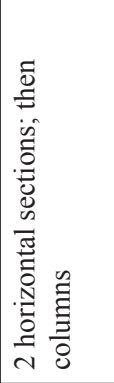 \\
\hline 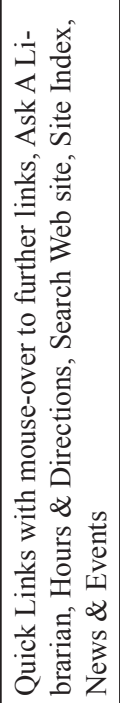 & 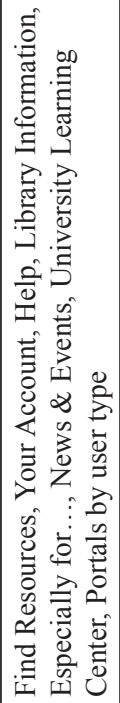 & 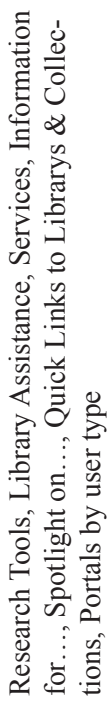 & 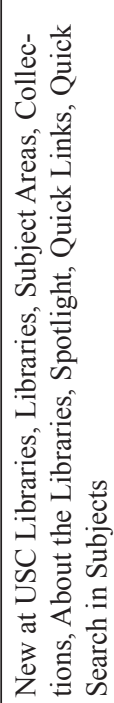 & 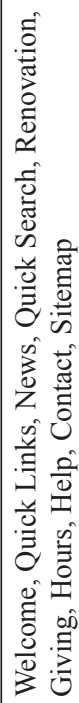 & 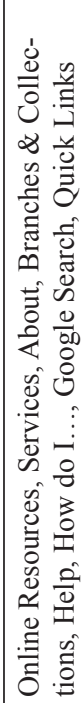 & 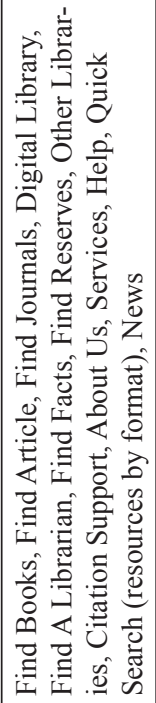 & 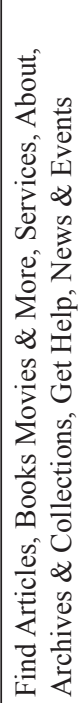 & 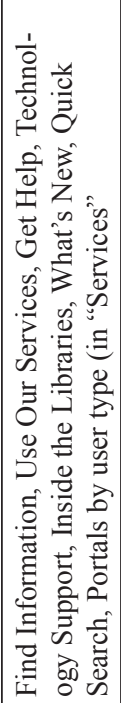 & 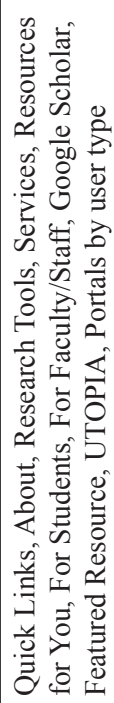 \\
\hline 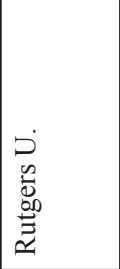 & 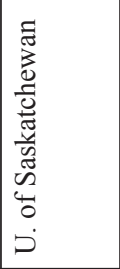 & 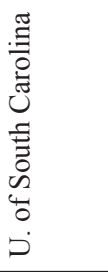 & 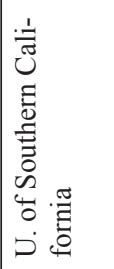 & 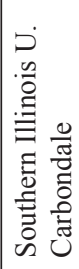 & 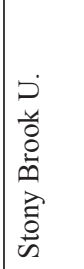 & 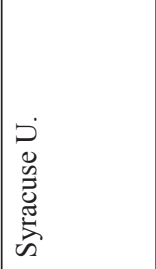 & 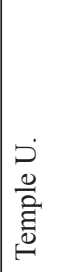 & 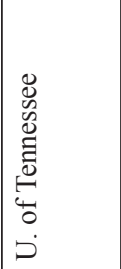 & 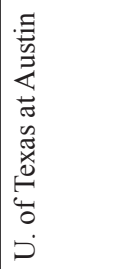 \\
\hline$\infty$ & $\infty$ & $\hat{\infty}$ & $\left.\right|_{\infty} ^{\infty}$ & $\infty$ & 18 & $\vec{a}$ & 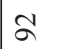 & $\hat{\sigma}$ & హ゙ \\
\hline
\end{tabular}


The Future of Academic Library Web Sites 25

\begin{tabular}{|c|c|c|c|c|c|c|c|c|c|}
\hline 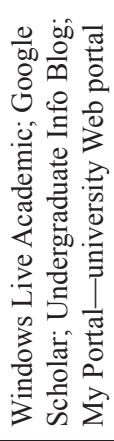 & 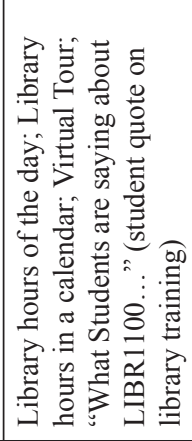 & 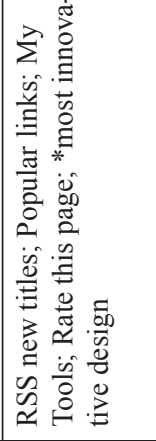 & & 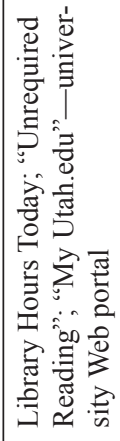 & 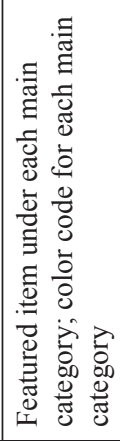 & 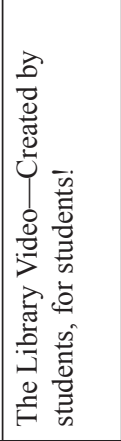 & 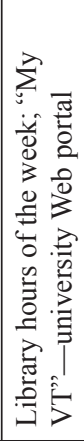 & & 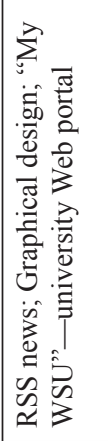 \\
\hline & 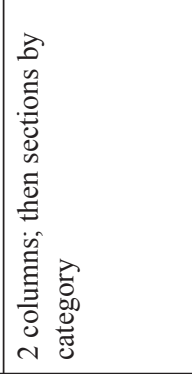 & 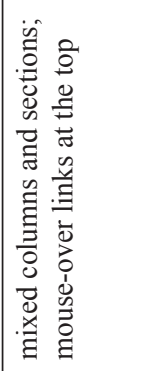 & 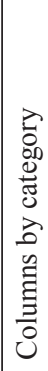 & 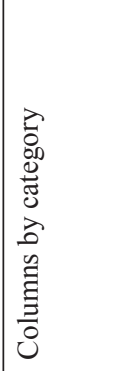 & 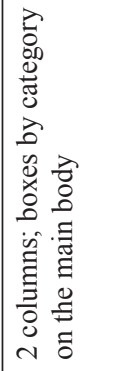 & 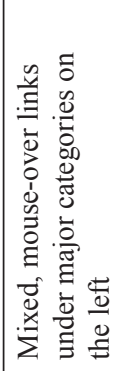 & 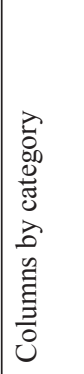 & 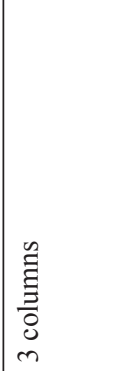 & 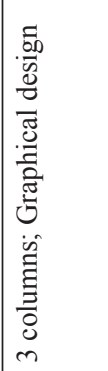 \\
\hline 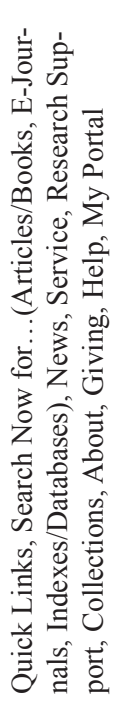 & 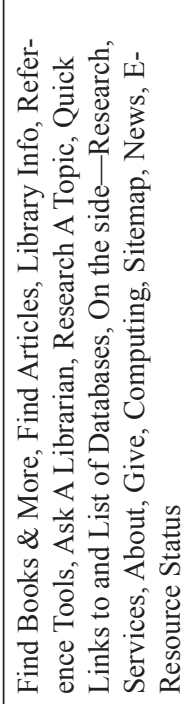 & 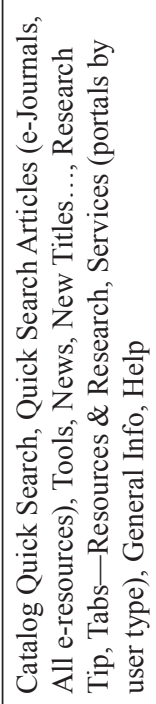 & 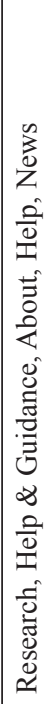 & 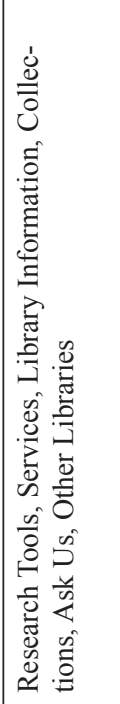 & 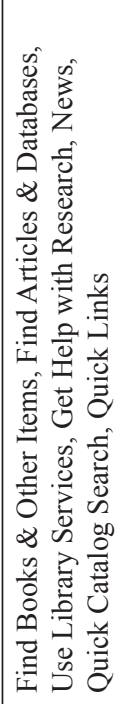 & 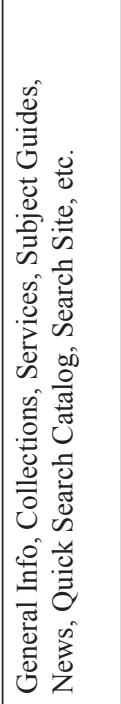 & 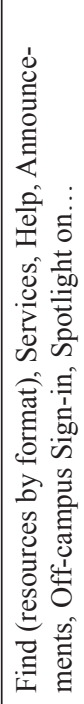 & 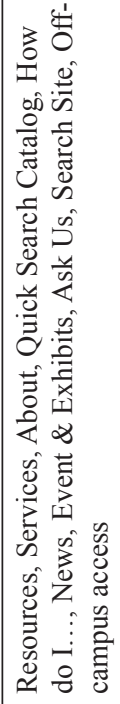 & 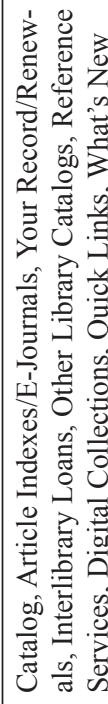 \\
\hline 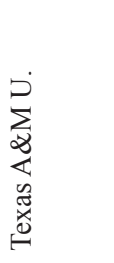 & 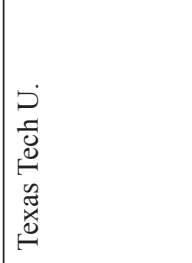 & 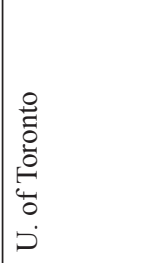 & 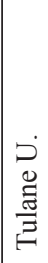 & 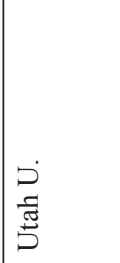 & 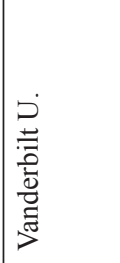 & 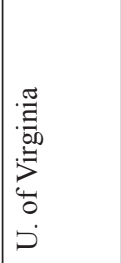 & 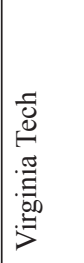 & 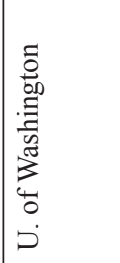 & 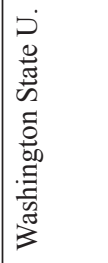 \\
\hline$a$ & $\curvearrowright$ & à & $\stackrel{\infty}{\infty}$ & - & 8 & $=$ & 0 & $=$ & $=$ \\
\hline
\end{tabular}




\begin{tabular}{|c|c|c|c|c|c|c|}
\hline $\begin{array}{l}0_{0}^{\infty} \\
0 \\
3 \\
0 \\
0 \\
0 \\
\text { है } \\
\sum^{2}\end{array}$ & & 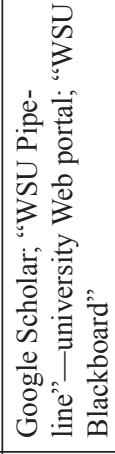 & 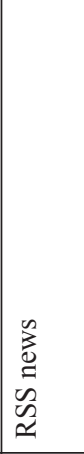 & 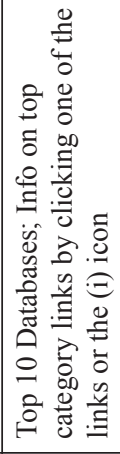 & 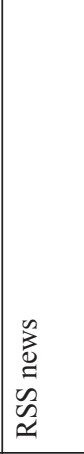 & 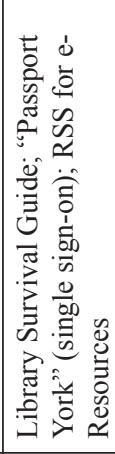 \\
\hline 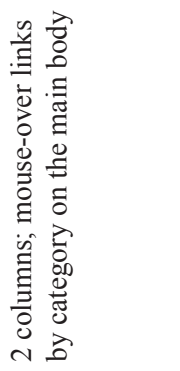 & 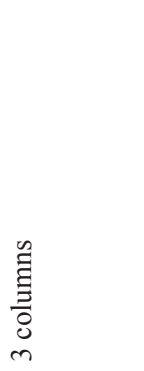 & 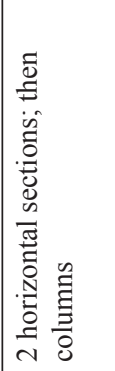 & 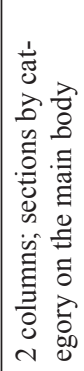 & 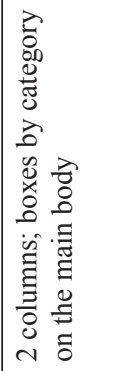 & 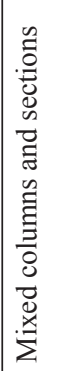 & 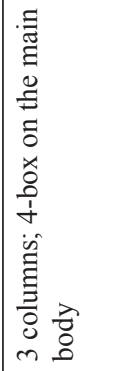 \\
\hline 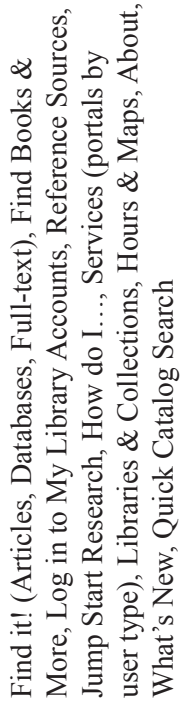 & 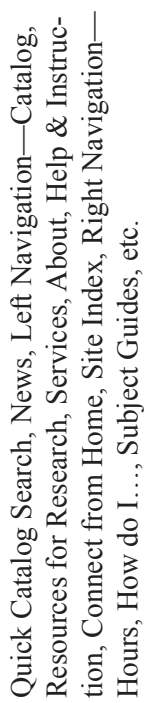 & 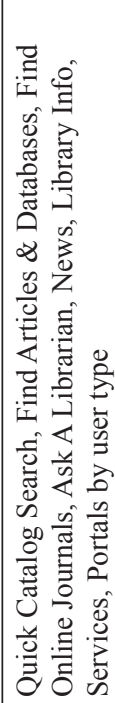 & 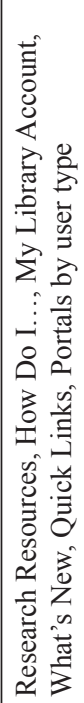 & 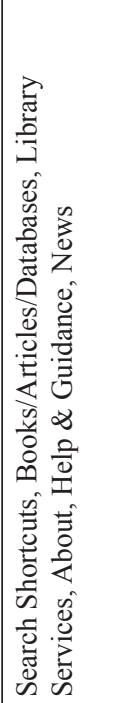 & 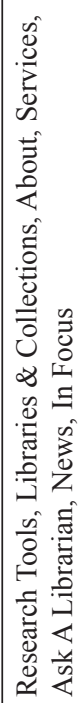 & 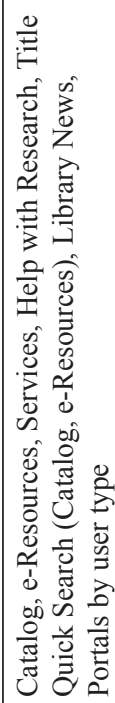 \\
\hline 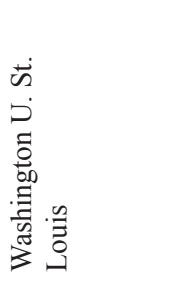 & 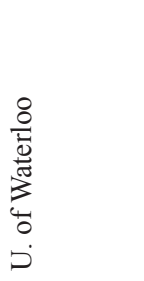 & 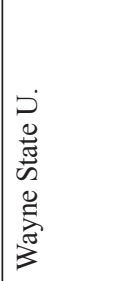 & 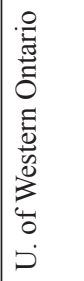 & 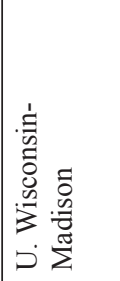 & 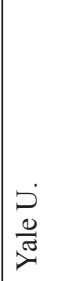 & $\begin{array}{l}0 \\
\ddot{0} \\
\overline{0}\end{array}$ \\
\hline 으 & $\stackrel{0}{=}$ & 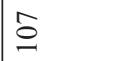 & 10 & 0 & $=$ & $=$ \\
\hline
\end{tabular}




\section{Appendix 2: Academic Library Web Sites That Employed RSS for Library News}

1. University at Albany, SUNY

2. University of Alberta

3. University of Arizona

4. Arizona State University

5. University at Buffalo, SUNY

6. Brigham Young University

7. University of California, Riverside

8. University of Guelph

9. Iowa State University

10. University of Kentucky

11. Massachusetts Institute of Technology

12. McGill University

13. McMaster University

14. University of Michigan

15. Michigan State University

16. University of Minnesota

17. University of North Carolina at Chapel Hill

18. North Carolina State University

19. Northwestern University

20. University of Oklahoma

21. Princeton University

22. Queen's University

23. Southern Illinois University Carbondale

24. Temple University

25. University of Tennessee

26. University of Texas at Austin

27. University of Toronto

28. Washington State University

29. Western Ontario University

30. Yale University 